\title{
PTSA-Catalyzed Reaction of Alkyl/Aryl Methyl Ketones with Aliphatic Alcohols in the Presence of Selenium Dioxide: A Protocol for the Generation of an $\alpha$-Ketoacetals Library
}

O. Risuklang Shangpliang, ${ }^{\dagger}$ Kmendashisha Wanniang, ${ }^{\dagger}$ Baskhemlang Kshiar, ${ }^{\dagger}$ Ibakyntiew D. Marpna, ${ }^{\dagger}$ Tyrchain Mitre Lipon, ${ }^{\dagger}$ Pushpak Mizar, ${ }^{\ddagger}$ and Bekington Myrboh* ${ }^{*},^{\dagger}$

${ }^{\dagger}$ Centre for Advanced Studies in Chemistry, Department of Chemistry, North-Eastern Hill University, Mawlai, Shillong 793022, India

${ }^{\ddagger}$ University of Southampton, Southampton SO17 1BJ, U.K.

Supporting Information

ABSTRACT: A novel approach has been developed for the synthesis of a wide range of $\alpha$-ketoacetals by the reaction of alkyl/aryl methyl ketones and aliphatic alcohols in the presence of selenium dioxide catalyzed by $p$-toluenesufonic acid. This method represents a general route to obtain a wide variety of $\alpha$-ketoacetals in a simple, rapid, and practical manner. This approach is particularly attractive because of the easy availability of the starting materials, mild reaction temperature, and good yields of the products. The resulting $\alpha$-ketoacetals are of much synthetic value as organic intermediates.

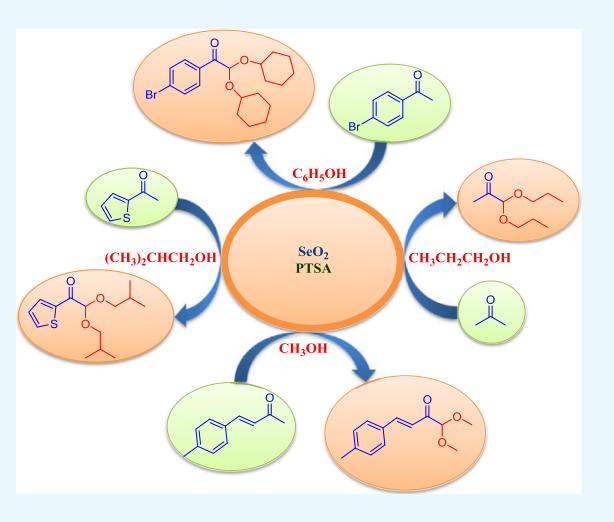

\section{INTRODUCTION}

The $\alpha$-ketoacetals are important functional moieties and are useful building blocks in organic synthesis. They are useful intermediates in that they provide an array of functional groups that are extremely valuable in organic syntheses. For instance the $\alpha$-ketoacetals are a key intermediate in the synthesis of various biological active compounds such as chiral $\alpha$-hydroxy acetals, ${ }^{1}$ chiral $\alpha$-amino acetals, ${ }^{2}$ chiral auxiliaries, ${ }^{3}$ and cyanosilylation ${ }^{4}$ and also for the construction of important heterocycles. ${ }^{5}$ Several methods have been described for the preparation of $\alpha$-ketoacetals. ${ }^{5-9}$ Goswami et al., reported the synthesis of aliphatic $\alpha$-ketoacetals starting from ketones via a two step procedure using $\mathrm{SeO}_{2} \cdot{ }^{5 \mathrm{a}, \mathrm{b}}$ Tiecco and co-workers reported the synthesis of $\alpha$-ketoacetals catalyzed by diphenyldiselenide and an excess of ammonium peroxydisulfate under reflux conditions (Scheme 1a). ${ }^{7}$ Ayala-Mata and group employed Weinreb amides as a starting material for the synthesis of $\alpha$-ketoacetals (Scheme $1 b$ ).$^{8}$ More recently, we have reported the synthesis of phenylglyoxal diethylacetals via the reaction of aromatic ketones with triethylorthoformate in the presence of $\mathrm{H}_{2} \mathrm{SeO}_{3}$ catalyzed by $\mathrm{BF}_{3} \cdot \mathrm{Et}_{2} \mathrm{O}$ (Scheme 1c). ${ }^{9}$ This method, though simple is limited by the use of triethylorthoformate as the sole source of alkoxide nucleophile. Generally, most of the other reported methods involved multistep reactions. Besides, the high cost of the reagents coupled with sensitive reaction conditions limit their scope of applications. Development of an alternative method for the synthesis of $\alpha$-ketoacetals with wide substrate scope starting
Scheme 1. Synthesis of $\alpha$-Ketoacetals

Previous Work

$$
\text { (a) }
$$

Our Work

$$
\text { 至 }
$$

from simple and easily available starting materials would therefore be a welcome addition to synthetic organic chemists.

The reactive behavior of $\mathrm{SeO}_{2}$ toward organic substrates in the presence of a Lewis acid or a strong organic acid, however, has found few or no mention at all in the literature. Earlier we

Received: February 13, 2019

Accepted: February 26, 2019

Published: March 29, 2019 
reported an efficient regio-selective protocol for the $\mathrm{C}-\mathrm{C}$ bond formation by an unexpected $\alpha, \alpha$-diarylation of aromatic ketones in the presence of selenium dioxide, catalyzed by boron trifluoride etherate. ${ }^{10}$ This unusual reactivity of $\mathrm{SeO}_{2}$ toward aromatic ketones in the presence of a Lewis acid prompted us to explore its reactions with organic substrates by varying the nature of the acid and the solvent used. Since then, we have further demonstrated this unique reactivity of $\mathrm{SeO}_{2}$ with aromatic ketones by changing the acid catalyst and/or the solvent used, leading to the formation of important organic intermediates. $^{11}$

As part of our on-going investigation on the synthetic utility of selenium dioxide, ${ }^{9-11}$ we wish to report here an efficient general method for the synthesis of $\alpha$-ketoacetals from a wide range of ketones and alcohols in presence of $\mathrm{SeO}_{2}$ catalyzed by p-toluenesufonic acid (PTSA) (Scheme 1).

\section{RESULTS AND DISCUSSION}

Initially, when a mixture of 1-(3-nitrophenyl)ethanone (1h) ( $1.0 \mathrm{mmol})$, ethanol $(\mathbf{2 a})(1 \mathrm{~mL}), \mathrm{SeO}_{2}$ (0.5 equiv), and PTSA ( 0.5 equiv) was stirred at room temperature for $12 \mathrm{~h}$, only a trace amount of the product was formed as observed by thinlayer chromatography (TLC) (Table 1, entry 1). When the

Table 1. Optimization of the Reaction Conditions ${ }^{a}$

\begin{tabular}{|c|c|c|c|c|c|}
\hline & h & $2 a$ & & & \\
\hline entry & $\begin{array}{l}\text { oxidant } \\
\text { (equiv) }\end{array}$ & $\begin{array}{l}\text { catalyst } \\
\text { (equiv) }\end{array}$ & $\begin{array}{l}\text { temperature } \\
{ }^{\circ} \mathrm{C}\end{array}$ & $t(\mathrm{~h})$ & $\begin{array}{c}\text { yield } \\
(\%)\end{array}$ \\
\hline 1 & $\mathrm{SeO}_{2}(0.5)$ & PTSA (0.5) & $\mathrm{rt}$ & 12 & trace \\
\hline 2 & $\mathrm{SeO}_{2}(0.5)$ & PTSA $(0.5)$ & 60 & 8 & 52 \\
\hline 3 & $\mathrm{SeO}_{2}(0.5)$ & PTSA (1.0) & 60 & 12 & 61 \\
\hline 4 & $\mathrm{SeO}_{2}(0.7)$ & PTSA (1.0) & 60 & 8 & 85 \\
\hline 5 & $\mathrm{SeO}_{2}(0.7)$ & PTSA (1.0) & 80 & 8 & 83 \\
\hline 6 & $\mathrm{SeO}_{2}(1.0)$ & PTSA (1.0) & 60 & 8 & 86 \\
\hline
\end{tabular}

${ }^{a}$ Reaction conditions: ketones $(1)(1.0 \mathrm{mmol})$, ethanol $(2)(1 \mathrm{~mL})$.

reaction temperature was raised to $60{ }^{\circ} \mathrm{C}$ for $8 \mathrm{~h}$, the yield increased to $52 \%$ (Table 1, entry 2). Any further increase in the temperature did not increase the yield any further. Further optimization reactions by varying the number of equivalents of selenium dioxide and PTSA (Table 1, entry 2-6) were carried out. It was found that ( 0.7 equiv) of $\mathrm{SeO}_{2}$ and ( 1.0 equiv) of PTSA gave optimum yield of the product (85\%) (Table 1, entry 4). To establish whether PTSA is unique to this reaction, other organic acids such as TFA and $\mathrm{CH}_{3} \mathrm{COOH}$ were employed and in both cases the desired product was not formed. It may be noted that the use of either $\mathrm{SeO}_{2}$ or PTSA alone failed to give the desired product.

With the optimized conditions in hand, the scope and generality of the reaction of aryl methyl ketones with alcohols were investigated. First, the reaction demonstrated a wide substrate scope in terms of the aromatic ketone (1) with ethanol (2a) (Scheme 2). Aryl methyl ketones bearing electron-neutral (4-H), electron-donating [e.g., 4-Me, 4-OH, 4-OMe, and 3,4-( $\mathrm{OMe})_{2}$ ] or electron-withdrawing (e.g., 3$\mathrm{NO}_{2}$ and 4- $\mathrm{NO}_{2}$ ) substituent in the ring were successfully converted to the corresponding products $\mathbf{3 a}-\mathbf{b}, \mathbf{3 e}-\mathbf{f}$, and $\mathbf{3 g}-$ i in moderate-to-good yields (59-90\%). The procedure was also found to be compatible with halogenated aryl methyl ketone (4-Br, 4-Cl), which gave the desired products in good yields (3c, 71\%; 3d, 74\%). The scope of the reaction was also extended to sterically hindered 2-acetyl naphthalene (1j), which readily yielded product $3 \mathbf{j}$ in $78 \%$ yield.

Second, homologs of alcohol from the aliphatic series (Scheme 2) were randomly selected. Both primary and secondary aliphatic alcohols easily undergo double nucleophillic attack on the $\alpha$-carbon atom of the ketone to give the desired products $3 \mathbf{k}-\mathbf{w}$ in moderate-to-good yields $(60-89 \%)$. Methanol (2b), 1-propanol (2c), 1-butanol (2d), 1-hexanol (2e), benzyl alcohol (2f), and iso-butanol $(\mathbf{2 g})$ readily reacted with aryl methyl ketones bearing electron-withdrawing group (3- $\left.\mathrm{NO}_{2}\right)$, electron-donating groups $\left[3,4-(\mathrm{OMe})_{2}\right]$, and halogenated aryl methyl ketones $(4-\mathrm{Br}, 4-\mathrm{Cl})$ to give the corresponding products $3 \mathbf{k}-\mathbf{p}$ and $3 \mathbf{s}-\mathbf{t}$ in moderate-to-good yields $(60-88 \%)$. Ordinarily one would have expected that long-chain aliphatic alcohol would not react because of steric consideration. Surprisingly however, the reaction with cetyl alcohol $(\mathbf{2 h})$ proceeded cleanly in $8 \mathrm{~h}$ to give excellent yields of the desired product $(3 \mathbf{v}, 85 \% ; 3 \mathbf{w}, 89 \%)$. Similarly, secondary alcohols such as iso-propanol (2i) and cyclohexanol $(2 \mathbf{j})$ also reacted smoothly to give the corresponding products $3 q-\mathbf{r}$ and $3 \mathbf{u}$ in satisfactory yields $(62-79 \%)$. However, tertiary aliphatic alcohol (2k) failed to react evidently because of the bulky nature of the substituent adjacent to the reacting nucleophile $3 \mathbf{x}$.

The methodology was further extended to the reaction of hetero-aryl methyl ketones (4) with aliphatic alcohol (2) which effortlessly gave the desired products $\mathbf{5 a}-\mathbf{d}$ in moderateto-good yields (72-93\%) (Scheme 3).

To further explore the efficacy of the method, reactions of substituted benzylidine acetones (6) with alcohols (2) were performed (Scheme 4). Benzylidine acetone bearing electron neutral (4-H), electron donating (e.g., 4-Me and 4-OMe), electron withdrawing $\left(4-\mathrm{NO}_{2}\right)$, or halogenated group (4-Br) all gave the expected products $7 \mathbf{a}-\mathbf{e}$ in good yield (82-92\%).

Having met with unprecedented success in the above reactions, we finally turned to the aliphatic ketones $\mathbf{8}$. We were delighted to note that the method continues to hold good with representative examples of cyclic and acyclic aliphatic ketones (Scheme 5). Reactions with aliphatic ketones such as acetone (8a), ethyl methyl ketone $(\mathbf{8 b})$, and pentanone (8c) proceeded as expected to give the corresponding products $9 \mathbf{a}-\mathbf{d}$ in moderate yield $(62-70 \%)$. Reactions with branched aliphatic ketones such as iso-butyl methyl ketone (8d) and isopentyl methyl ketone $(\mathbf{8 e})$ also gave the desired products $(9 \mathbf{e}$, $69 \%$; 9f, 67\%) in good yields. Similarly, secondary aliphatic ketones 3-methyl-2-butanone (8f) and the strained cyclopropyl ketone $(\mathbf{8 g})$ afforded the desired products $9 \mathrm{~g}$ and $9 \mathrm{~h}$ in 65 and $73 \%$ yields respectively.

Previously, we had proposed the reactions to proceed via the intermediate 10 (Scheme 6) where precipitation of elemental selenium occurred. In the present work, particularly, the precipitation of elemental selenium was clean with no formation of colloidal Se. Although we have not succeeded in isolating the intermediate $\mathbf{1 0}$ so far, evidently the mechanism follows the same route as reported in our previous work (Scheme 6).,10,11c

\section{CONCLUSION}

We have developed a simple and efficient approach for the synthesis of $\alpha$-ketoacetals from aryl/alkyl methyl ketones with 
Scheme 2. Scope of Aryl Methyl Ketones That Couple with Alcohols ${ }^{a}$

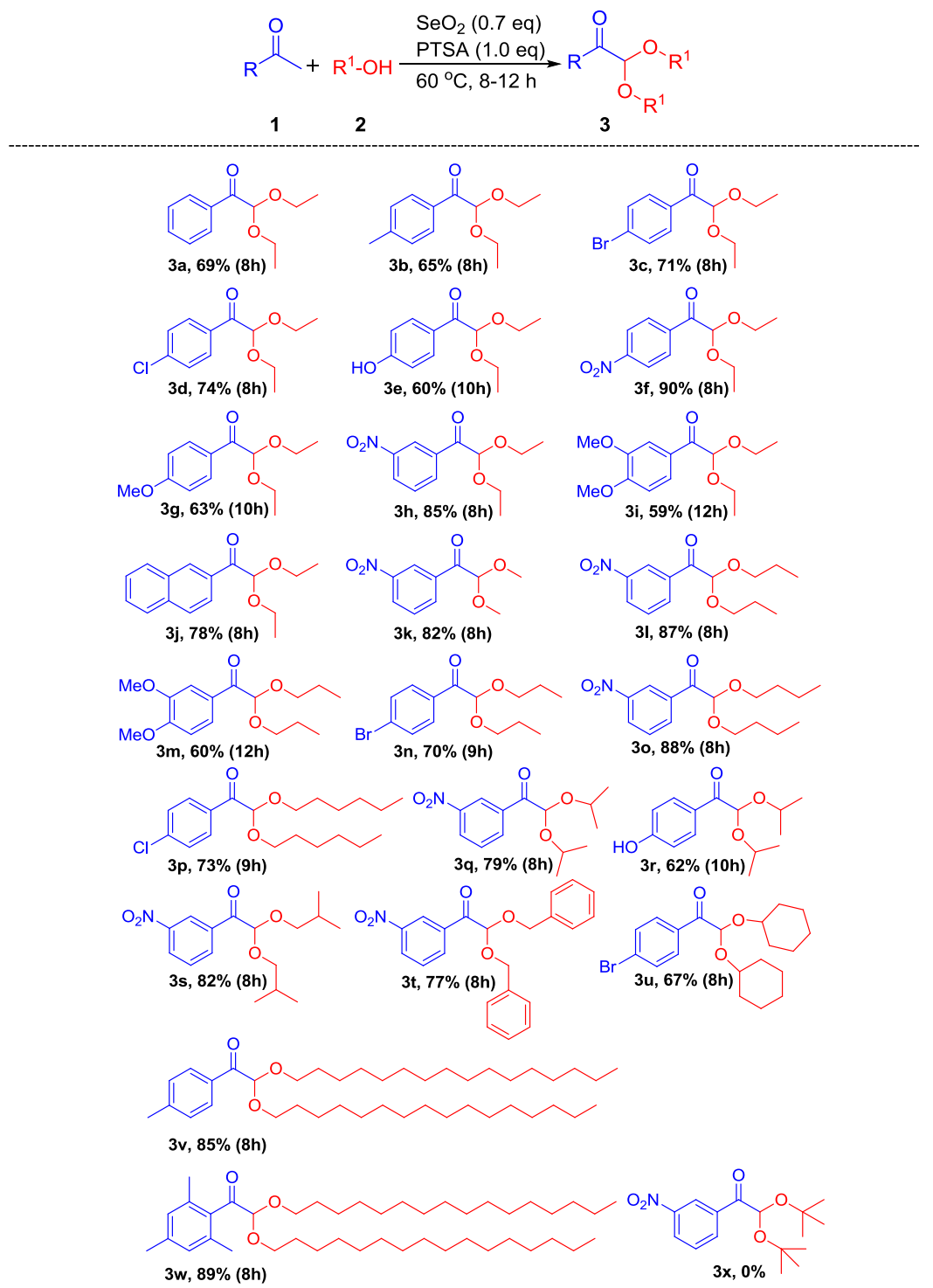

${ }^{a}$ Reaction conditions: ketones $(1)(1.0 \mathrm{mmol})$, alcohols $(2)(1 \mathrm{~mL}), \mathrm{SeO}_{2}\left(0.7\right.$ equiv), PTSA (1.0 equiv) at $60{ }^{\circ} \mathrm{C}, 8-12 \mathrm{~h}$.

Scheme 3. Scope of Heteroaryl Methyl Ketones That Couple with Alcohols ${ }^{a}$
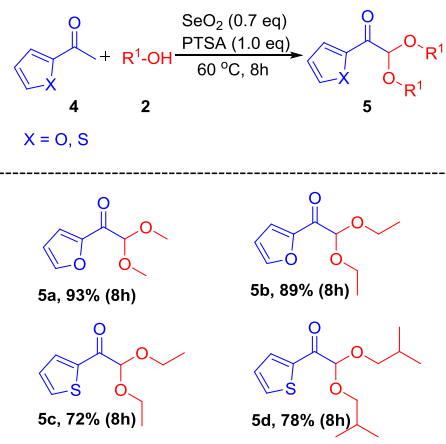

${ }^{a}$ Reaction conditions: ketones (4) (1.0 mmol), alcohol (2) (1 mL), $\mathrm{SeO}_{2}$ (0.7 equiv), PTSA (1.0 equiv) at $60{ }^{\circ} \mathrm{C}, 8 \mathrm{~h}$.

aliphatic alcohols in the presence of selenium dioxide and PTSA. The methodology further demonstrates its generality for a diversity-oriented synthesis of novel $\alpha$-ketoacetals library.
Scheme 4. Scope of $\alpha, \beta$-Unsaturated Ketones That Couple with Alcohols ${ }^{a}$

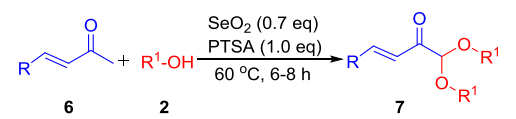

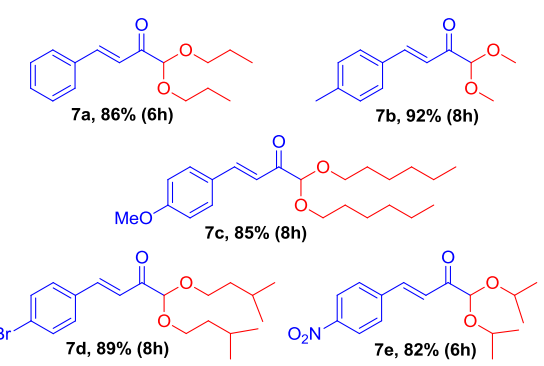

${ }^{a}$ Reaction conditions: ketones $(6)(1.0 \mathrm{mmol})$, alcohols $(2)(1 \mathrm{~mL})$, $\mathrm{SeO}_{2}$ (0.7 equiv), PTSA ( 1.0 equiv) at $60^{\circ} \mathrm{C}, 6-8 \mathrm{~h}$. 
Scheme 5. Scope of Aliphatic Ketones That Couple with Alcohols $^{a}$

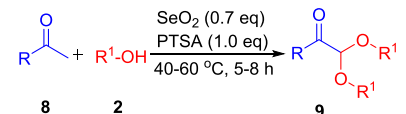

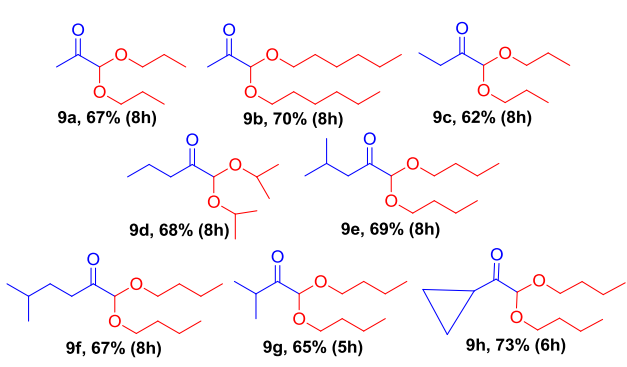

${ }^{a}$ Reaction conditions: ketones (8) $(1.0 \mathrm{mmol})$, alcohol (2) (1 mL), $\mathrm{SeO}_{2}$ (0.7 equiv), PTSA (1.0 equiv), $40-60{ }^{\circ} \mathrm{C}, 5-8 \mathrm{~h}$.

\section{Scheme 6. Plausible Mechanism}
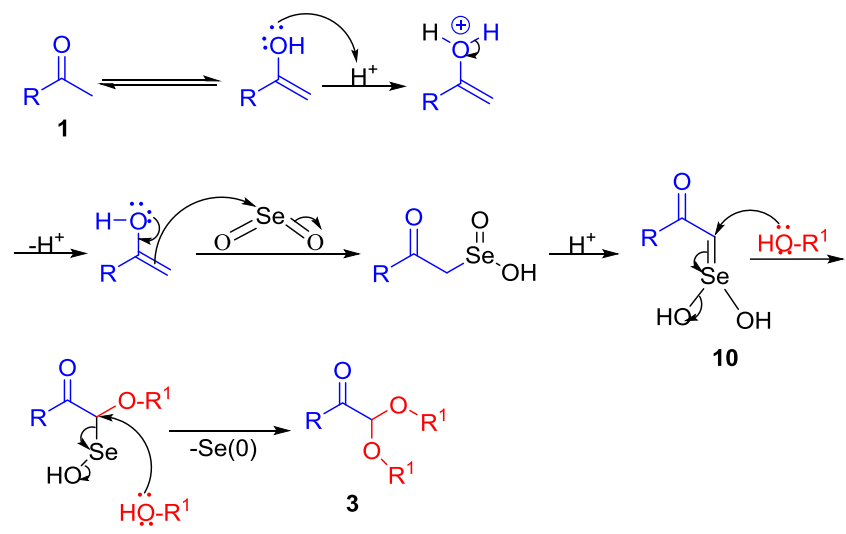

The reactions exhibited wide substrate tolerance in both the reactants. The easy availability of the reactants used coupled with the simplicity of the reaction procedure involved will certainly make this methodology a more attractive and viable alternative.

\section{EXPERIMENTAL SECTION}

General Methods. All reagents were purchased from Sigma-Aldrich, TCI Chemicals, and Alfa Aesar and were used without further purification unless noted. Melting points were recorded by the open capillary tube method and are uncorrected. IR spectra were recorded on a PerkinElmer Spectrum 400 FTIR instrument. ${ }^{1} \mathrm{H}$ NMR and ${ }^{13} \mathrm{C}\left\{{ }^{1} \mathrm{H}\right\}$ NMR spectra were recorded on a Bruker Avance II-400 spectrometer in $\mathrm{CDCl}_{3}$ with TMS as the internal standard. Mass spectral data were obtained with a Waters UPLC-TQD mass spectrometer (ESI-MS). Elemental analyses were carried out on PerkinElmer 2400 Series II. High-resolution mass spectra (ESI-HRMS) were recorded on a MaXis (Bruker Daltonics, Bremen, Germany) time of flight mass spectrometer. All reactions were monitored by TLC using precoated aluminium sheets (silica gel 60 F254 $0.2 \mathrm{~mm}$ thickness). Column chromatography was carried out on silica gel (100-200 mesh), and flash chromatography was carried out on silica gel (230-400 mesh).

General Procedure for the Synthesis of Compounds $3,5,7$, and 9. A mixture of the aromatic ketone 1 or 4 or $\alpha$, $\beta$-unsaturated ketones 6 or aliphatic ketones $8(1.0 \mathrm{mmol})$, selenium dioxide (77 mg, $0.7 \mathrm{mmol}, 0.7$ equiv), PTSA (190 $\mathrm{mg}, 1.0 \mathrm{mmol}, 1.0$ equiv), and alcohol $2(1 \mathrm{~mL})$ was allowed to stir at $40-60{ }^{\circ} \mathrm{C}$ for $5-12 \mathrm{~h}$. Then, a thick precipitate of elemental selenium settled at the bottom of the flask which was then filtered off, washed with ethyl acetate $(2 \times 10 \mathrm{~mL})$, and the combined filtrate was transferred to a separating funnel, washed with conc. sodium bicarbonate solution $(2 \times 10 \mathrm{~mL})$ followed by brine $(2 \times 10 \mathrm{~mL})$. The organic layer was separated, dried over anhydrous $\mathrm{Na}_{2} \mathrm{SO}_{4}$, and concentrated in vacuo. The crude mass was purified by column chromatography using silica gel (100-200 mesh) or flash chromatography using silica gel (230-400 mesh) and ethyl acetatehexane as the eluent.

2,2-Diethoxy-1-phenylethanone $(3 a) .^{9}$ The title compound was prepared via the general procedure from acetophenone 1a (120 mg, $1.0 \mathrm{mmol})$ and ethanol 2a (1 $\mathrm{mL}$ ). The product was isolated by flash chromatography using $1 \%$ ethyl acetate/hexane as an eluent; oil (143 mg, 69\% yield); IR (KBr film): 3064, 2979, 2883, 1686, 1599, 1449, 1360, 1266, 1119, $1062 \mathrm{~cm}^{-1}$; ${ }^{1} \mathrm{H}$ NMR (400 MHz, $\left.\mathrm{CDCl}_{3}\right): \delta$ 8.17-7.43 (m, 5H), $5.29(\mathrm{~s}, 1 \mathrm{H}), 3.80-3.64(\mathrm{~m}, 4 \mathrm{H}), 1.25(\mathrm{t}$, $J=7.2 \mathrm{~Hz}, 6 \mathrm{H}) \mathrm{ppm} ;{ }^{13} \mathrm{C}\left\{{ }^{1} \mathrm{H}\right\} \mathrm{NMR}\left(100 \mathrm{MHz}, \mathrm{CDCl}_{3}\right): \delta$ 194.0, 133.7, 133.4, 129.7, 128.3, 102.3, 63.1, 15.2 ppm. MS $\left(\mathrm{ES}^{+}\right)$: for $\mathrm{C}_{12} \mathrm{H}_{16} \mathrm{O}_{3}, 208.1$; found $m / z, 231.2[\mathrm{M}+\mathrm{Na}]^{+}$.

2,2-Diethoxy-1-(p-tolyl)ethanone (3b). ${ }^{9}$ The title compound was prepared via the general procedure from 4-methylacetophenone $\mathbf{1 b}(134 \mathrm{mg}, 1.0 \mathrm{mmol})$ and ethanol $\mathbf{2 a}(1 \mathrm{~mL})$. The product was isolated by flash chromatography using $1 \%$ ethyl acetate/hexane as an eluent; oil (144 mg, 65\% yield); IR (KBr film): 3030, 3004, 2922, 2870, 1682, 1606, 1574, 1428, $1405,1358,1268,1181,1122,1018 \mathrm{~cm}^{-1} ;{ }^{1} \mathrm{H}$ NMR (400 $\left.\mathrm{MHz}, \mathrm{CDCl}_{3}\right): \delta 7.99(\mathrm{~d}, J=7.2 \mathrm{~Hz}, 2 \mathrm{H}), 7.18(\mathrm{~d}, J=8.8 \mathrm{~Hz}$, $2 \mathrm{H}), 5.21(\mathrm{~s}, 1 \mathrm{H}), 3.71-3.56(\mathrm{~m}, 4 \mathrm{H}), 2.34(\mathrm{~s}, 3 \mathrm{H}) 1.17(\mathrm{t}, J$ $=7.2 \mathrm{~Hz} 6 \mathrm{H}) \mathrm{ppm} ;{ }^{13} \mathrm{C}\left\{{ }^{1} \mathrm{H}\right\} \mathrm{NMR}\left(100 \mathrm{MHz}, \mathrm{CDCl}_{3}\right): \delta$ $192.6,143.3,130.2,128.8,128.0,101.2,62.0,20.7,14.1 \mathrm{ppm}$. MS $\left(\mathrm{ES}^{+}\right)$: for $\mathrm{C}_{13} \mathrm{H}_{18} \mathrm{O}_{3}, 222.1$; found $m / z, 245.1[\mathrm{M}+\mathrm{Na}]^{+}$.

1-(4-Bromophenyl)-2,2-diethoxyethanone (3c). ${ }^{9}$ The title compound was prepared via the general procedure from 4bromo-acetophenone 1c (199 mg, $1.0 \mathrm{mmol})$ and ethanol 2a $(1 \mathrm{~mL})$. The product was isolated by flash chromatography using $1 \%$ ethyl acetate/hexane as an eluent; oil (203 mg, 71\% yield); IR (KBr film): 3094, 2979, 2931, 2883, 1693, 1585, 1484, 1400, 1287, 1118, 1070, $1011 \mathrm{~cm}^{-1} ;{ }^{1} \mathrm{H}$ NMR $(400$ $\left.\mathrm{MHz}, \mathrm{CDCl}_{3}\right): \delta 7.97(\mathrm{~d}, J=8.8 \mathrm{~Hz}, 2 \mathrm{H}), 7.51(\mathrm{~d}, J=8.4 \mathrm{~Hz}$, $2 \mathrm{H}), 5.10(\mathrm{~s}, 1 \mathrm{H}), 3.73-3.53(\mathrm{~m}, 4 \mathrm{H}), 1.16(\mathrm{t}, J=7.2 \mathrm{~Hz}, 6 \mathrm{H})$ ppm; ${ }^{13} \mathrm{C}\left\{{ }^{1} \mathrm{H}\right\}$ NMR $\left(100 \mathrm{MHz}, \mathrm{CDCl}_{3}\right): \delta$ 193.2, 132.2, 131.6, 131.4, 128.7, 103.0, 63.5, $15.2 \mathrm{ppm}$. MS $\left(\mathrm{ES}^{+}\right)$: for $\mathrm{C}_{12} \mathrm{H}_{15} \mathrm{BrO}_{3}, 286.0$; found $m / z, 309.1[\mathrm{M}+\mathrm{Na}]^{+}$.

1-(4-Chlorophenyl)-2,2-diethoxyethanone (3d). ${ }^{9}$ The title compound was prepared via the general procedure from 4chloro-acetophenone $\mathbf{1 d}(154 \mathrm{mg}, 1.0 \mathrm{mmol})$ and ethanol 2a $(1 \mathrm{~mL})$. The product was isolated by flash chromatography using $1 \%$ ethyl acetate/hexane as an eluent; oil $(178 \mathrm{mg}, 74 \%$ yield); IR ( KBr film): 3077, 2980, 2931, 2883, 1693, 1589, 1570, 1488, 1403, 1286, 1200, 1092, $1015 \mathrm{~cm}^{-1}$; ${ }^{1} \mathrm{H}$ NMR $\left(400 \mathrm{MHz}, \mathrm{CDCl}_{3}\right): \delta 8.05(\mathrm{~d}, J=9.6 \mathrm{~Hz}, 2 \mathrm{H}), 7.35$ (d, $J=8.4$ $\mathrm{Hz}, 2 \mathrm{H}), 5.10(\mathrm{~s}, 1 \mathrm{H}), 3.73-3.54(\mathrm{~m}, 4 \mathrm{H}), 1.17(\mathrm{t}, J=7.6 \mathrm{~Hz}$, $6 \mathrm{H}) \mathrm{ppm} ;{ }^{13} \mathrm{C}\left\{{ }^{1} \mathrm{H}\right\} \mathrm{NMR}\left(100 \mathrm{MHz}, \mathrm{CDCl}_{3}\right): \delta 193.0,139.9$, $131.8,131.3,128.6,103.0,63.5,15.1 \mathrm{ppm} . \mathrm{MS}\left(\mathrm{ES}^{+}\right)$: for $\mathrm{C}_{12} \mathrm{H}_{15} \mathrm{ClO}_{3}, 242.0$; found $m / z, 265.2[\mathrm{M}+\mathrm{Na}]^{+}$.

2,2-Diethoxy-1-(4-hydroxyphenyl)ethanone (3e). ${ }^{9}$ The title compound was prepared via the general procedure from 
4-hydroy-acetophenone $1 \mathrm{e}(136 \mathrm{mg}, 1.0 \mathrm{mmol})$ and ethanol 2a $(1 \mathrm{~mL})$. The product was isolated by flash chromatography using $2 \%$ ethyl acetate/hexane as an eluent; oil (134 mg, 60\% yield); IR ( $\mathrm{KBr}$ film): 3330, 2982, 2935, 2896, 1675, 1602, $1515,1443,1372,1290,1164,1056 \mathrm{~cm}^{-1} ;{ }^{1} \mathrm{H}$ NMR (400 $\left.\mathrm{MHz} \mathrm{CDCl}_{3}\right): \delta 8.00(\mathrm{~d}, J=8.4 \mathrm{~Hz}, 2 \mathrm{H}), 7.65(\mathrm{~s} \mathrm{br}, 1 \mathrm{H}), 6.83$ $(\mathrm{d}, J=8.4 \mathrm{~Hz}, 2 \mathrm{H}), 5.28(\mathrm{~s}, 1 \mathrm{H}), 3.68-3.53(\mathrm{~m}, 4 \mathrm{H}), 1.15(\mathrm{t}, J$ $=7.2 \mathrm{~Hz}, 6 \mathrm{H}) \mathrm{ppm} ;{ }^{13} \mathrm{C}\left\{{ }^{1} \mathrm{H}\right\} \mathrm{NMR}\left(100 \mathrm{MHz}, \mathrm{CDCl}_{3}\right): \delta$ 193.4, 161.8, 132.4, 126.0, 115.5, 101.2, 62.8, 15.1 ppm. MS $\left(\mathrm{ES}^{+}\right)$: for $\mathrm{C}_{12} \mathrm{H}_{16} \mathrm{O}_{4}, 224.1$; found $m / z, 225.0[\mathrm{M}+\mathrm{H}]^{+}$.

2,2-Diethoxy-1-(4-nitrophenyl)ethanone (3f). ${ }^{9}$ The title compound was prepared via the general procedure from 4nitro-acetophenone if (165 mg, $1.0 \mathrm{mmol})$ and ethanol 2a (1 $\mathrm{mL}$ ). The product was isolated by flash chromatography using $5 \%$ ethyl acetate/hexane as an eluent; oil $(227 \mathrm{mg}, 90 \%$ yield $)$; IR ( $\mathrm{KBr}$ film): 3107, 3051, 2978, 2939, 2903, 1702, 1603, $1528,1481,1370,1345,1329,1278,1109,1053,1017 \mathrm{~cm}^{-1}$; ${ }^{1} \mathrm{H} \mathrm{NMR}\left(400 \mathrm{MHz}, \mathrm{CDCl}_{3}\right): \delta 8.28(\mathrm{~d}, J=9.6 \mathrm{~Hz}, 2 \mathrm{H}), 8.21$ $(\mathrm{d}, J=8.4 \mathrm{~Hz}, 2 \mathrm{H}), 5.07(\mathrm{~s}, 1 \mathrm{H}), 3.77-3.54(\mathrm{~m}, 4 \mathrm{H}), 1.18(\mathrm{t}, J$ $=7.2 \mathrm{~Hz}, 6 \mathrm{H}) \mathrm{ppm} ;{ }^{13} \mathrm{C}\left\{{ }^{1} \mathrm{H}\right\} \mathrm{NMR}\left(100 \mathrm{MHz}, \mathrm{CDCl}_{3}\right): \delta$ 192.7, 150.3, 138.0, 131.0, 123.3, 103.7, 64.1, 15.1 ppm. MS $\left(\mathrm{ES}^{+}\right)$: for $\mathrm{C}_{12} \mathrm{H}_{15} \mathrm{NO}_{5}, 253.1$; found $m / z, 276.1[\mathrm{M}+\mathrm{Na}]^{+}$.

2,2-Diethoxy-1-(4-methoxyphenyl)ethanone (3g). ${ }^{9}$ The title compound was prepared via the general procedure from 4-methoxy-acetophenone $1 \mathrm{~g}(150 \mathrm{mg}, 1.0 \mathrm{mmol})$ and ethanol 2a $(1 \mathrm{~mL})$. The product was isolated by flash chromatography using $1 \%$ ethyl acetate/hexane as an eluent; oil (149 mg, 63\% yield); IR ( $\mathrm{KBr}$ film): 2978, 2935, 2896, 2844, 1679, 1601, $1575,1511,1460,1422,1308,1260,1173,1114,1062,1029$ $\mathrm{cm}^{-1} ;{ }^{1} \mathrm{H}$ NMR (400 MHz, $\left.\mathrm{CDCl}_{3}\right): \delta 8.08(\mathrm{~d}, J=8.8 \mathrm{~Hz}$, $2 \mathrm{H}), 6.85(\mathrm{~d}, J=8.4 \mathrm{~Hz}, 2 \mathrm{H}), 5.17(\mathrm{~s}, 1 \mathrm{H}), 3.80(\mathrm{~s}, 3 \mathrm{H})$, $3.71-3.55(\mathrm{~m}, 4 \mathrm{H}), 1.17(\mathrm{t}, J=7.6 \mathrm{~Hz}, 6 \mathrm{H}) \mathrm{ppm} ;{ }^{13} \mathrm{C}\left\{{ }^{1} \mathrm{H}\right\}-$ NMR $\left(100 \mathrm{MHz}, \mathrm{CDCl}_{3}\right): \delta 192.7,163.7,132.1,130.6,113.5$, 102.5, 63.0, 55.4, 15.2 ppm. MS $\left(\mathrm{ES}^{+}\right)$: for $\mathrm{C}_{13} \mathrm{H}_{18} \mathrm{O}_{4}, 238.1$; found $m / z, 261.1[\mathrm{M}+\mathrm{Na}]^{+}$.

2,2-Diethoxy-1-(3-nitrophenyl)ethanone (3h). ${ }^{9}$ The title compound was prepared via the general procedure from 3nitro-acetophenone $1 \mathrm{~h}(165 \mathrm{mg}, 1.0 \mathrm{mmol})$ and ethanol $\mathbf{2 a}$ (1 $\mathrm{mL}$ ). The product was isolated by flash chromatography using $5 \%$ ethyl acetate/hexane as an eluent; oil $(215 \mathrm{mg}, 85 \%$ yield); IR ( $\mathrm{KBr}$ film): 3088, 2980, 2935, 2885, 1703, 1614, 1580, 1534, 1478, 1441, 1351, 1271, 1228, $1062 \mathrm{~cm}^{-1} ;{ }^{1} \mathrm{H}$ NMR $\left(400 \mathrm{MHz}, \mathrm{CDCl}_{3}\right): \delta 8.96(\mathrm{~s}, 1 \mathrm{H}), 8.43(\mathrm{~d}, J=8.8 \mathrm{~Hz}, 1 \mathrm{H})$, $8.34(\mathrm{~d}, J=6.0 \mathrm{~Hz}, 1 \mathrm{H}), 7.58(\mathrm{t}, J=8.8 \mathrm{~Hz}, 1 \mathrm{H}), 5.09(\mathrm{~s}, 1 \mathrm{H})$, $3.80-3.57(\mathrm{~m}, 4 \mathrm{H}), 1.19(\mathrm{t}, J=7.2 \mathrm{~Hz}, 6 \mathrm{H}) \mathrm{ppm} ;{ }^{13} \mathrm{C}\left\{{ }^{1} \mathrm{H}\right\}-$ NMR $\left(100 \mathrm{MHz}, \mathrm{CDCl}_{3}\right): \delta 192.0,148.1,135.6,134.6,129.4$, $127.5,125.0,103.5,64.1,15.1 \mathrm{ppm}$. MS $\left(\mathrm{ES}^{+}\right)$: for $\mathrm{C}_{12} \mathrm{H}_{15} \mathrm{NO}_{5}, 253.1$; found $m / z, 276.1[\mathrm{M}+\mathrm{Na}]^{+}$.

2,2-Diethoxy-1-(3,4-dimethoxyphenyl)ethanone (3i). The title compound was prepared via the general procedure from 3,4-dimethoxy-acetophenone $1 \mathrm{i}(180 \mathrm{mg}, 1.0 \mathrm{mmol})$ and ethanol 2a $(1 \mathrm{~mL})$. The product was isolated by flash chromatography using 1\% ethyl acetate/hexane as an eluent; oil (158 mg, 59\% yield); IR (KBr film): 2977, 2935, 2844, $1681,1595,1515,1464,1421,1343,1273,1229,1174,1121$, $1060,1023 \mathrm{~cm}^{-1}$; ${ }^{1} \mathrm{H}$ NMR $\left(400 \mathrm{MHz}, \mathrm{CDCl}_{3}\right): \delta 7.84(\mathrm{~d}, J=$ $8.8 \mathrm{~Hz}, 1 \mathrm{H}), 7.59(\mathrm{~s}, 1 \mathrm{H}), 6.82(\mathrm{~d}, J=8.8 \mathrm{~Hz}, 1 \mathrm{H}), 5.19(\mathrm{~s}$, $1 \mathrm{H}), 3.88(\mathrm{~s}, 3 \mathrm{H}), 3.86(\mathrm{~s}, 3 \mathrm{H}), 3.72-3.54(\mathrm{~m}, 4 \mathrm{H}), 1.18(\mathrm{t}, J$ $=6.0 \mathrm{~Hz}, 6 \mathrm{H}) \mathrm{ppm} ;{ }^{13} \mathrm{C}\left\{{ }^{1} \mathrm{H}\right\} \mathrm{NMR}\left(100 \mathrm{MHz}, \mathrm{CDCl}_{3}\right): \delta$ 192.6, 153.5, 148.7, 126.7, 124.8, 111.4, 109.9, 102.4, 63.0, 56.0, 55.9, 15.2 ppm. MS (ES ${ }^{+}$: for $\mathrm{C}_{14} \mathrm{H}_{20} \mathrm{O}_{5}, 268.1$; found $m / z, 269.7[\mathrm{M}+\mathrm{H}]^{+}$. HRMS (ESI) $m / z:[\mathrm{M}+\mathrm{Na}]^{+}$calcd for $\mathrm{C}_{14} \mathrm{H}_{20} \mathrm{O}_{5} \mathrm{Na}$, 291.1208; found, 291.1209.
2,2-Diethoxy-1-(naphthalen-2-yl)ethanone (3j). ${ }^{9}$ The title compound was prepared via the general procedure from 2 acetylnaphthalene $\mathbf{1 j}(170 \mathrm{mg}, 1.0 \mathrm{mmol})$ and ethanol 2a (1 $\mathrm{mL})$. The product was isolated by flash chromatography using $1 \%$ ethyl acetate/hexane as an eluent; oil (201 mg, 78\% yield); IR (KBr film): 3059, 2977, 2882, 1681, 1627, 1597, 1468, 1438, 1361, 1281, 1115, $1061 \mathrm{~cm}^{-1}$; ${ }^{1} \mathrm{H}$ NMR (400 MHz, $\left.\mathrm{CDCl}_{3}\right): \delta 8.71(\mathrm{~s}, 1 \mathrm{H}), 8.08-7.46(\mathrm{~m}, 6 \mathrm{H}), 5.34(\mathrm{~s}, 1 \mathrm{H})$, $3.76-3.60(\mathrm{~m}, 4 \mathrm{H}), 1.19(\mathrm{t}, J=7.2 \mathrm{~Hz}, 6 \mathrm{H}) \mathrm{ppm} ;{ }^{13} \mathrm{C}\left\{{ }^{1} \mathrm{H}\right\}-$ NMR (100 MHz, $\left.\mathrm{CDCl}_{3}\right): \delta$ 194.0, 135.8, 132.0, 129.9, 129.5, 128.6, 128.4, 128.1, 127.7, 126.6, 124.9, 102.4, 63.1, 15.2 ppm. MS $\left(\mathrm{ES}^{+}\right)$: for $\mathrm{C}_{16} \mathrm{H}_{18} \mathrm{O}_{3}, 258.1$; found $m / z, 281.0[\mathrm{M}+\mathrm{Na}]^{+}$.

2,2-Dimethoxy-1-(3-nitrophenyl)ethanone (3k). The title compound was prepared via the general procedure from 3nitro-acetophenone $1 \mathrm{~h}(165 \mathrm{mg}, 1.0 \mathrm{mmol})$ and methanol $\mathbf{2 b}$ $(1 \mathrm{~mL})$. The product was isolated by flash chromatography using $2 \%$ ethyl acetate/hexane as an eluent; oil $(184 \mathrm{mg}, 82 \%$ yield); IR ( $\mathrm{KBr}$ film): 3088, 2942, 2837, 1697, 1615, 1532, 1351, 1192, $1093 \mathrm{~cm}^{-1}$; ${ }^{1} \mathrm{H}$ NMR (400 MHz, $\left.\mathrm{CDCl}_{3}\right): \delta 8.90$ $(\mathrm{s}, 1 \mathrm{H}), 8.40-8.35(\mathrm{~m}, 2 \mathrm{H}), 7.60(\mathrm{t}, J=7.6 \mathrm{~Hz}, 1 \mathrm{H}), 5.03(\mathrm{~s}$, $1 \mathrm{H}), 3.46(\mathrm{~s}, 6 \mathrm{H}) \mathrm{ppm} ;{ }^{13} \mathrm{C}\left\{{ }^{1} \mathrm{H}\right\} \mathrm{NMR}\left(100 \mathrm{MHz}, \mathrm{CDCl}_{3}\right): \delta$ 191.5 , 148.2, 135.4, 134.6, 129.6, 127.7, 124.8, 104.8, 55.4 ppm. MS $\left(\mathrm{ES}^{+}\right)$: for $\mathrm{C}_{10} \mathrm{H}_{11} \mathrm{NO}_{5}, 225.0$; found $\mathrm{m} / z, 248.0[\mathrm{M}$ $+\mathrm{Na}]^{+}$.

2,2-Dipropoxy-1-(3-nitrophenyl)ethanone (3I). The title compound was prepared via the general procedure from 3nitro-acetophenone $1 \mathrm{~h}(165 \mathrm{mg}, 1.0 \mathrm{mmol})$ and 1-propanol $2 \mathrm{c}$ $(1 \mathrm{~mL})$. The product was isolated by flash chromatography using 5\% ethyl acetate/hexane as an eluent; oil (244 mg, 87\% yield); IR ( $\mathrm{KBr}$ film): 3120, 2967, 2937, 2878, 1702, 1615, $1534,1477,1438,1350,1300,1267,1191,1122,1100,1071$ $\mathrm{cm}^{-1}$; ${ }^{1} \mathrm{H}$ NMR $\left(400 \mathrm{MHz}, \mathrm{CDCl}_{3}\right): \delta 8.97(\mathrm{~s}, 1 \mathrm{H}), 8.43$ (d, $J$ $=7.2 \mathrm{~Hz}, 1 \mathrm{H}), 8.34(\mathrm{~d}, J=6.0 \mathrm{~Hz}, 1 \mathrm{H}), 7.58(\mathrm{t}, J=7.6 \mathrm{~Hz}$, $1 \mathrm{H}), 5.05(\mathrm{~s}, 1 \mathrm{H}), 3.69-3.45(\mathrm{~m}, 4 \mathrm{H}), 1.62-1.53(\mathrm{~m}, 4 \mathrm{H})$, $0.84(\mathrm{t}, J=7.2 \mathrm{~Hz}, 6 \mathrm{H}) \mathrm{ppm} ;{ }^{13} \mathrm{C}\left\{{ }^{1} \mathrm{H}\right\} \mathrm{NMR}(100 \mathrm{MHz}$, $\left.\mathrm{CDCl}_{3}\right): \delta 192.1,148.1,135.6,134.6,129.4,127.5,125.1$, 104.2, 70.4, 22.9, 10.5 ppm. MS $\left(\mathrm{ES}^{+}\right)$: for $\mathrm{C}_{14} \mathrm{H}_{19} \mathrm{NO}_{5}, 281.1$; found $m / z, 299.0\left[\mathrm{M}+\mathrm{NH}_{4}\right]^{+}$. HRMS (ESI) $m / z:[\mathrm{M}+\mathrm{Na}]^{+}$ calcd for $\mathrm{C}_{14} \mathrm{H}_{19} \mathrm{NO}_{5} \mathrm{Na}, 304.1161$; found, 304.1158.

1-(3,4-Dimethoxyphenyl)-2,2-dipropoxyethanone (3m). The title compound was prepared via the general procedure from 3,4-dimethoxy-acetophenone $\mathbf{1 h}(180 \mathrm{mg}, 1.0 \mathrm{mmol})$ and 1-propanol 2c $(1 \mathrm{~mL})$. The product was isolated by flash chromatography using 3\% ethyl acetate/hexane as an eluent; oil (177 mg, 60\% yield); IR (KBr film): 2964, 2939, 2877, $1680,1595,1515,1464,1421,1344,1273,1228,1119,1069$, $1024 \mathrm{~cm}^{-1}$; ${ }^{1} \mathrm{H}$ NMR $\left(400 \mathrm{MHz}, \mathrm{CDCl}_{3}\right): \delta 7.84(\mathrm{~d}, J=8.4$ $\mathrm{Hz}, 1 \mathrm{H}), 7.61(\mathrm{~s}, 1 \mathrm{H}), 6.82(\mathrm{~d}, J=8.4 \mathrm{~Hz}, 1 \mathrm{H}), 5.14(\mathrm{~s}, 1 \mathrm{H})$, $3.88(\mathrm{~s}, 3 \mathrm{H}), 3.86(\mathrm{~s}, 3 \mathrm{H}), 3.61-3.43(\mathrm{~m}, 4 \mathrm{H}), 1.61-1.52(\mathrm{~m}$, $4 \mathrm{H}), 0.84(\mathrm{t}, J=7.2 \mathrm{~Hz}, 6 \mathrm{H}) \mathrm{ppm} ;{ }^{13} \mathrm{C}\left\{{ }^{1} \mathrm{H}\right\} \mathrm{NMR}(100 \mathrm{MHz}$, $\left.\mathrm{CDCl}_{3}\right): \delta 191.7,152.5,147.6,125.7,123.9,110.6,108.9$, 102.2, 68.4, 55.0, 54.9, 21.9, 9.5 ppm. MS $\left(\mathrm{ES}^{+}\right)$: for $\mathrm{C}_{16} \mathrm{H}_{24} \mathrm{O}_{5}$, 296.1; found $m / z, 297.2[\mathrm{M}+\mathrm{H}]^{+}$. HRMS (ESI) $\mathrm{m} / z$ : $[\mathrm{M}+$ $\mathrm{Na}]^{+}$calcd for $\mathrm{C}_{16} \mathrm{H}_{24} \mathrm{O}_{5} \mathrm{Na}$, 319.1521; found, 319.1523 .

1-(4-Bromophenyl)-2,2-dipropoxyethanone (3n). The title compound was prepared via the general procedure from 4bromo-acetophenone $1 \mathrm{c}(199 \mathrm{mg}, 1.0 \mathrm{mmol})$ and 1-propanol $2 \mathrm{c}(1 \mathrm{~mL})$. The product was isolated by flash chromatography using $1 \%$ ethyl acetate/hexane as an eluent; oil $(220 \mathrm{mg}, 70 \%$ yield); IR (KBr film): 2965, 2935, 2877, 1690, 1586, 1483, 1396, 1265, 1116, 1071, $1010 \mathrm{~cm}^{-1}$; ${ }^{1} \mathrm{H}$ NMR $(400 \mathrm{MHz}$, $\left.\mathrm{CDCl}_{3}\right): \delta 8.04(\mathrm{~d}, J=8.8 \mathrm{~Hz}, 2 \mathrm{H}), 7.59(\mathrm{~d}, J=9.2 \mathrm{~Hz}, 2 \mathrm{H})$, $5.13(\mathrm{~s}, 1 \mathrm{H}), 3.69-3.48(\mathrm{~m}, 4 \mathrm{H}), 1.67-1.58(\mathrm{~m}, 4 \mathrm{H}), 0.90(\mathrm{t}$, 
$J=7.6 \mathrm{~Hz}, 6 \mathrm{H}) \mathrm{ppm} ;{ }^{13} \mathrm{C}\left\{{ }^{1} \mathrm{H}\right\} \mathrm{NMR}\left(100 \mathrm{MHz}, \mathrm{CDCl}_{3}\right): \delta$ 192.2 , 131.2, 130.5, 130.4, 127.6, 102.7, 68.8, 21.8, 9.5 ppm. HRMS (ESI) $m / z:[\mathrm{M}+\mathrm{Na}]^{+}$calcd for $\mathrm{C}_{14} \mathrm{H}_{19} \mathrm{BrO}_{3} \mathrm{Na}$, 337.0415; found, 337.0411 .

2,2-Dibutoxy-1-(3-nitrophenyl)ethanone (30). The title compound was prepared via the general procedure from 3nitro-acetophenone $1 \mathrm{~h}(165 \mathrm{mg}, 1.0 \mathrm{mmol})$ and 1-butanol $2 \mathrm{~d}$ $(1 \mathrm{~mL})$. The product was isolated by flash chromatography using 5\% ethyl acetate/hexane as an eluent; oil (271 mg, $88 \%$ yield); IR ( $\mathrm{KBr}$ film): 3087, 2960, 2935, 2874, 1702, 1614, 1580, 1534, 1466, 1350, 1299, 1269, 1228, 1125, $1073 \mathrm{~cm}^{-1}$; ${ }^{1} \mathrm{H} \mathrm{NMR}\left(400 \mathrm{MHz}, \mathrm{CDCl}_{3}\right): \delta 8.97(\mathrm{~s}, 1 \mathrm{H}), 8.42(\mathrm{~d}, J=7.2$ $\mathrm{Hz}, 1 \mathrm{H}), 8.34(\mathrm{~d}, J=6.0 \mathrm{~Hz}, 1 \mathrm{H}), 7.58(\mathrm{t}, J=7.2 \mathrm{~Hz}, 1 \mathrm{H})$, $5.03(\mathrm{~s}, 1 \mathrm{H}), 3.73-3.48(\mathrm{~m}, 4 \mathrm{H}), 1.56-1.49(\mathrm{~m}, 4 \mathrm{H}), 1.33-$ $1.24(\mathrm{~m}, 4 \mathrm{H}), 0.82(\mathrm{t}, J=7.2 \mathrm{~Hz}, 6 \mathrm{H}) \mathrm{ppm} ;{ }^{13} \mathrm{C}\left\{{ }^{1} \mathrm{H}\right\} \mathrm{NMR}$ $\left(100 \mathrm{MHz}, \mathrm{CDCl}_{3}\right): \delta 192.1,148.1,135.6,134.6,129.4,127.5$, $125.1,104.3,68.5,31.6,19.1,13.7 \mathrm{ppm} . \mathrm{MS}\left(\mathrm{ES}^{+}\right)$: for $\mathrm{C}_{16} \mathrm{H}_{23} \mathrm{NO}_{5}, 309.1$; found $\mathrm{m} / z, 309.0[\mathrm{M}]^{+}$. HRMS (ESI) $\mathrm{m} / z$ : $[\mathrm{M}+\mathrm{Na}]^{+}$calcd for $\mathrm{C}_{16} \mathrm{H}_{23} \mathrm{NO}_{5} \mathrm{Na}, 332.1474$; found, 332.1736 .

1-(4-Chlorophenyl)-2,2-bis (hexyloxy)ethanone (3p). The title compound was prepared via the general procedure from 4chloro-acetophenone 1d (154 mg, $1.0 \mathrm{mmol})$ and 1-hexanol 2e $(1 \mathrm{~mL})$. The product was isolated by flash chromatography using $1 \%$ ethyl acetate/hexane as an eluent; oil $(258 \mathrm{mg}, 73 \%$ yield); IR ( $\mathrm{KBr}$ film): 3136, 2956, 2932, 2861, 1694, 1589, 1401, 1385, 1284, 1192, 1121, $1092 \mathrm{~cm}^{-1}$; ${ }^{1} \mathrm{H}$ NMR (400 $\left.\mathrm{MHz}, \mathrm{CDCl}_{3}\right): \delta 8.04(\mathrm{~d}, J=8.4 \mathrm{~Hz}, 2 \mathrm{H}), 7.34(\mathrm{~d}, J=8.4 \mathrm{~Hz}$, $2 \mathrm{H}), 5.04(\mathrm{~s}, 1 \mathrm{H}), 3.64-3.44(\mathrm{~m}, 4 \mathrm{H}), 1.56-1.49(\mathrm{~m}, 4 \mathrm{H})$, $1.27-1.15(\mathrm{~m}, 12 \mathrm{H}), 0.78(\mathrm{t}, J=6.8 \mathrm{~Hz}, 6 \mathrm{H}) \mathrm{ppm} ;{ }^{13} \mathrm{C}\left\{{ }^{1} \mathrm{H}\right\}-$ NMR $\left(100 \mathrm{MHz}, \mathrm{CDCl}_{3}\right): \delta 193.1,139.8,131.8,131.3,128.5$, 103.6, 68.1, 31.4, 29.5, 25, 6, 22.5, 13.9 ppm; $M S\left(\mathrm{ES}^{+}\right)$: for $\mathrm{C}_{20} \mathrm{H}_{31} \mathrm{ClO}_{3}, 354.2$; found $m / z, 377.3[\mathrm{M}+\mathrm{Na}]^{+}$. HRMS (ESI) $m / z:[\mathrm{M}+\mathrm{Na}]^{+}$calcd for $\mathrm{C}_{20} \mathrm{H}_{31} \mathrm{ClO}_{3} \mathrm{Na}, 377.1859$; found, 377.1856 .

2,2-Diisopropoxy-1-(3-nitrophenyl)ethanone (3q). The title compound was prepared via the general procedure from 3-nitro-acetophenone $1 \mathrm{~h}(165 \mathrm{mg}, 1.0 \mathrm{mmol}$ ) and isopropanol $2 \mathbf{i}(1 \mathrm{~mL})$. The product was isolated by flash chromatography using 5\% ethyl acetate/hexane as an eluent; oil (222 mg, 79\% yield); IR (KBr film): 3088, 2977, 2934, $2892,1703,1615,1580,1535,1467,1438,1378,1351,1320$, $1269,1180,1122,1099,1034 \mathrm{~cm}^{-1} ;{ }^{1} \mathrm{H}$ NMR $(400 \mathrm{MHz}$, $\left.\mathrm{CDCl}_{3}\right): \delta 9.01(\mathrm{~s}, 1 \mathrm{H}), 8.48-8.31(\mathrm{~m}, 2 \mathrm{H}), 7.56(\mathrm{t}, J=7.2$ $\mathrm{Hz}, 1 \mathrm{H}), 5.07(\mathrm{~s}, 1 \mathrm{H}), 3.94-3.85(\mathrm{~m}, 2 \mathrm{H}), 1.22(\mathrm{~d}, J=6.0 \mathrm{~Hz}$, $6 \mathrm{H}) 1.07(\mathrm{~d}, J=6.0 \mathrm{~Hz}, 6 \mathrm{H}) \mathrm{ppm} ;{ }^{13} \mathrm{C}\left\{{ }^{1} \mathrm{H}\right\} \mathrm{NMR}(100 \mathrm{MHz}$, $\left.\mathrm{CDCl}_{3}\right): \delta 192.7,148.0,136.1,134.4,129.2,127.3,125.5$, 102.2, 71.0, 22.9, 22.2 ppm; MS (ES $)$ : for $\mathrm{C}_{14} \mathrm{H}_{19} \mathrm{NO}_{5}, 281.1$; found $m / z, 299\left[\mathrm{M}+\mathrm{NH}_{4}\right]^{+}$. HRMS (ESI) $m / z:[\mathrm{M}+\mathrm{Na}]^{+}$ calcd for $\mathrm{C}_{14} \mathrm{H}_{19} \mathrm{NO}_{5} \mathrm{Na}, 304.1161$; found, 304.1162.

1-(4-Hydroxyphenyl)-2,2-diisopropoxyethanone (3r). The title compound was prepared via the general procedure from 4hydroxy-acetophenone $1 \mathrm{e}(136 \mathrm{mg}, 1.0 \mathrm{mmol})$ and isopropanol $2 \mathrm{i}(1 \mathrm{~mL})$. The product was isolated by flash chromatography using 5\% ethyl acetate/hexane as an eluent; oil (156 mg, 62\% yield); IR (KBr film): 3339, 2975, 2933, $1674,1602,1582,1515,1443,1438,1383,1288,1241,1171$, $1119,1038 \mathrm{~cm}^{-1}$; ${ }^{1} \mathrm{H}$ NMR $\left(400 \mathrm{MHz}, \mathrm{CDCl}_{3}\right): \delta 8.15(\mathrm{~d}, J=$ $8.8 \mathrm{~Hz}, 2 \mathrm{H}), 6.87(\mathrm{~d}, J=8.8 \mathrm{~Hz}, 2 \mathrm{H}), 5.20(\mathrm{~s}, 1 \mathrm{H}), 3.97-3.90$ $(\mathrm{m}, 12 \mathrm{H}), 1.24(\mathrm{~d}, J=6.0 \mathrm{~Hz}, 6 \mathrm{H}) 1.14(\mathrm{~d}, J=6.0 \mathrm{~Hz}, 6 \mathrm{H})$ ppm; ${ }^{13} \mathrm{C}\left\{{ }^{1} \mathrm{H}\right\} \mathrm{NMR}\left(100 \mathrm{MHz}, \mathrm{CDCl}_{3}\right): \delta$ 194.6, 161.3, 133.2, 126.2, 115.4, 101.6, 70.5, 23.1, 22.5 ppm. MS (ES ${ }^{+}$): for $\mathrm{C}_{14} \mathrm{H}_{20} \mathrm{O}_{4}, 252.1$; found $m / z, 275.0[\mathrm{M}+\mathrm{Na}]^{+}$. HRMS (ESI) $m / z:[\mathrm{M}+\mathrm{Na}]^{+}$calcd for $\mathrm{C}_{14} \mathrm{H}_{20} \mathrm{O}_{4} \mathrm{Na}, 275.1259$; found, 275.1257 .

2,2-Diisobutoxy-1-(3-nitrophenyl)ethanone (3s). The title compound was prepared via the general procedure from 3nitro-acetophenone $\mathbf{1 h}(165 \mathrm{mg}, 1.0 \mathrm{mmol})$ and iso-butanol $\mathbf{2 g}$ $(1 \mathrm{~mL})$. The product was isolated by flash chromatography using 5\% ethyl acetate/hexane as an eluent; oil $(253 \mathrm{mg}, 82 \%$ yield); IR (KBr film): 3090, 2960, 2875, 1703, 1615, 1580, 1536, 1472, 1438, 1349, 1301, 1266, $1055 \mathrm{~cm}^{-1}$; ${ }^{1} \mathrm{H}$ NMR $\left(400 \mathrm{MHz}, \mathrm{CDCl}_{3}\right): \delta 9.00(\mathrm{~s}, 1 \mathrm{H}), 8.43(\mathrm{~d}, J=7.2 \mathrm{~Hz}, 1 \mathrm{H})$, $8.35-8.33(\mathrm{~m}, 1 \mathrm{H}), 7.58(\mathrm{t}, J=7.2 \mathrm{~Hz}, 1 \mathrm{H}), 4.99(\mathrm{~s}, 1 \mathrm{H})$, $3.50-3.25(\mathrm{~m}, 4 \mathrm{H}), 1.88-1.78(\mathrm{~m}, 2 \mathrm{H}), 0.83(\mathrm{t}, J=6 \mathrm{~Hz}$, $12 \mathrm{H}) \mathrm{ppm} ;{ }^{13} \mathrm{C}\left\{{ }^{1} \mathrm{H}\right\} \mathrm{NMR}\left(100 \mathrm{MHz}, \mathrm{CDCl}_{3}\right): \delta$ 192.0, 148.1, $135.6,134.6,129.3,127.5,125.2,104.8,75.5,28.5,19.2,19.1$ ppm. MS $\left(\mathrm{ES}^{+}\right)$: for $\mathrm{C}_{16} \mathrm{H}_{23} \mathrm{NO}_{5} 309.1$, found $m / z$, 327.1 [M + $\left.\mathrm{NH}_{4}\right]^{+}$. HRMS (ESI) $m / z:[\mathrm{M}+\mathrm{Na}]^{+}$calcd for $\mathrm{C}_{16} \mathrm{H}_{23} \mathrm{NO}_{5} \mathrm{Na}$, 332.3518; found, 332.3514 .

2,2-Bis(benzyloxy)-1-(3-nitrophenyl)ethanone (3t). ${ }^{4 C}$ The title compound was prepared via the general procedure from 3nitro-acetophenone $1 \mathrm{~h}(165 \mathrm{mg}, 1.0 \mathrm{mmol})$ and benzyl alcohol $2 \mathrm{f}(1 \mathrm{~mL})$. The product was isolated by flash chromatography using 5\% ethyl acetate/hexane as an eluent; oil $(290 \mathrm{mg}, 77 \%$ yield); IR (KBr film): 3088, 3034, 2931, 2874, 1698, 1614, $1580,1532,1497,1454,1350,1257,1124,1101 \mathrm{~cm}^{-1} ;{ }^{1} \mathrm{H}$ $\operatorname{NMR}\left(400 \mathrm{MHz}, \mathrm{CDCl}_{3}\right): \delta 8.93(\mathrm{~s}, 1 \mathrm{H}), 8.38-8.31(\mathrm{~m}, 2 \mathrm{H})$, $7.54(\mathrm{t}, J=7.6 \mathrm{~Hz}, 1 \mathrm{H}), 7.25(\mathrm{~s}, 5 \mathrm{H}), 7.24(\mathrm{~s}, 5 \mathrm{H}), 5.26(\mathrm{~s}$, $1 \mathrm{H}), 4.72-4.58(\mathrm{~m}, 4 \mathrm{H}) \mathrm{ppm} ;{ }^{13} \mathrm{C}\left\{{ }^{1} \mathrm{H}\right\} \mathrm{NMR}(100 \mathrm{MHz}$, $\left.\mathrm{CDCl}_{3}\right): \delta 191.6,148.1,136.3,135.5,134.6,129.5,128.6$, $128.3,128.2,127.6,125.0,101.6,70.0 \mathrm{ppm}$. MS $\left(\mathrm{ES}^{+}\right)$: for $\mathrm{C}_{22} \mathrm{H}_{19} \mathrm{NO}_{5}, 377.13$; found $m / z, 395.42\left[\mathrm{M}+\mathrm{NH}_{4}\right]^{+}$.

1-(4-Bromophenyl)-2,2-bis(cyclohexyloxy)ethanone (3u). The title compound was prepared via the general procedure from 4-bromo-acetophenone $1 \mathrm{c}(199 \mathrm{mg}, 1.0 \mathrm{mmol})$ and cyclohexanol $2 \mathrm{j}(1 \mathrm{~mL})$. The product was isolated by flash chromatography using $1 \%$ ethyl acetate/hexane as an eluent; off-white solid ( $264 \mathrm{mg}$, 67\% yield); mp $47-49{ }^{\circ} \mathrm{C}$; IR ( $\mathrm{KBr}$ film): 2933, 2856, 1689, 1585, 1449, 1281, 1117, $1038 \mathrm{~cm}^{-1}$; ${ }^{1} \mathrm{H}$ NMR $\left(400 \mathrm{MHz} \mathrm{CDCl}_{3}\right): \delta 8.03(\mathrm{~d}, J=8.8 \mathrm{~Hz}, 2 \mathrm{H}), 7.50$ $(\mathrm{d}, J=8.8 \mathrm{~Hz}, 2 \mathrm{H}), 5.10(\mathrm{~s}, 1 \mathrm{H}), 3.57-3.50(\mathrm{~m}, 2 \mathrm{H}), 1.85-$ $1.11(\mathrm{~m}, 20 \mathrm{H}) \mathrm{ppm} ;{ }^{13} \mathrm{C}\left\{{ }^{1} \mathrm{H}\right\} \mathrm{NMR}\left(100 \mathrm{MHz}, \mathrm{CDCl}_{3}\right): \delta$ 194.3, 132.0, 131.9, 131.4, 128.4, 102.2, 76.4, 32.9, 32.2, 25.4 ppm. HRMS (ESI) $m / z$ : $[\mathrm{M}+\mathrm{Na}]^{+}$calcd for $\mathrm{C}_{20} \mathrm{H}_{27} \mathrm{BrO}_{3} \mathrm{Na}$, 417.1041; found, 417.1034.

2,2-Bis(hexadecyloxy)-1-(p-tolyl)ethanone (3v). The title compound was prepared via the general procedure from 4methyl-acetophenone $\mathbf{1 b}(134 \mathrm{mg}, 1.0 \mathrm{mmol})$ and cetyl alcohol $2 \mathrm{~h}(0.5 \mathrm{~g})$. The product was isolated by flash chromatography using $1 \%$ ethyl acetate/hexane as an eluent; white solid( $522 \mathrm{mg}, 85 \%$ yield); mp $43-45{ }^{\circ} \mathrm{C}$; IR ( $\mathrm{KBr}$ film): 3054, 2955, 2917, 2850, 1689, 1605, 1471, 1242, 1132, 1041 $\mathrm{cm}^{-1}$; ${ }^{1} \mathrm{H}$ NMR $\left(400 \mathrm{MHz}, \mathrm{CDCl}_{3}\right): \delta 7.98(\mathrm{~d}, J=8.4 \mathrm{~Hz}$, $2 \mathrm{H}), 7.17(\mathrm{~d}, J=8 \mathrm{~Hz}, 2 \mathrm{H}), 5.15(\mathrm{~s}, 1 \mathrm{H}), 3.60-3.47(\mathrm{~m}, 4 \mathrm{H})$, $2.33(\mathrm{~s}, 3 \mathrm{H}), 1.56-1.49(\mathrm{~m}, 4 \mathrm{H}), 1.22-1.16(\mathrm{~m}, 52 \mathrm{H}), 0.80$ $(\mathrm{t}, J=6.8 \mathrm{~Hz}, 6 \mathrm{H}) \mathrm{ppm} ;{ }^{13} \mathrm{C}\left\{{ }^{1} \mathrm{H}\right\} \mathrm{NMR}\left(100 \mathrm{MHz}, \mathrm{CDCl}_{3}\right): \delta$ 193.8, 144.2, 131.2, 129.9, 129.0, 102.6, 67.6, 31.9, 29.72, 29.70, 29.68, 29.64, 29.61, 29.5, 29.38, 29.33, 26.0, 22.7, 21.7, $14.1 \mathrm{ppm}$. MS $\left(\mathrm{ES}^{+}\right)$: for $\mathrm{C}_{41} \mathrm{H}_{74} \mathrm{O}_{3}, 614.5$; found $\mathrm{m} / z, 615.8$ $[\mathrm{M}+\mathrm{H}]^{+}$. Anal. Calcd for $\mathrm{C}_{41} \mathrm{H}_{74} \mathrm{O}_{3}: \mathrm{C}, 80.07 ; \mathrm{H}, 12.13$; O, 7.80. Found: $\mathrm{C}, 80.21 ; \mathrm{H}, 12.35$; O, 7.75.

2,2-Bis(hexadecyloxy)-1-mesitylethanone (3w). The title compound was prepared via the general procedure from 2,4,6trimethyl-acetophenone 1c (162 mg, $1.0 \mathrm{mmol})$ and cetyl alcohol $2 \mathrm{~h}(0.5 \mathrm{~g})$. The product was isolated by flash 
chromatography using $1 \%$ ethyl acetate/hexane as an eluent; off-white solid (571 mg, 89\% yield); mp 29-31 ${ }^{\circ} \mathrm{C}$; IR ( $\mathrm{KBr}$ film): 2954, 2918, 2851, 1721, 1611, 1469, 1127, $1052 \mathrm{~cm}^{-1}$; ${ }^{1} \mathrm{H}$ NMR (400 MHz, $\left.\mathrm{CDCl}_{3}\right): \delta 6.73(\mathrm{~s}, 2 \mathrm{H}), 4.87(\mathrm{~s}, 1 \mathrm{H})$, $3.66-3.43(\mathrm{~m}, 4 \mathrm{H}), 2.19(\mathrm{~s}, 3 \mathrm{H}), 2.14(\mathrm{~s}, 6 \mathrm{H}), 1.54-1.47(\mathrm{~m}$, $4 \mathrm{H}), 1.24-1.18(\mathrm{~m}, 52 \mathrm{H}), 0.80(\mathrm{t}, J=6.8 \mathrm{~Hz}, 6 \mathrm{H}) \mathrm{ppm}$; ${ }^{13} \mathrm{C}\left\{{ }^{1} \mathrm{H}\right\} \mathrm{NMR}\left(100 \mathrm{MHz}, \mathrm{CDCl}_{3}\right): \delta$ 204.7, 138.5, 136.7, 133.9, 128.2, 102.2, 68.1, 31.9, 29.72, 29.70, 29.69, 29.67, 29.64, 29.61, 29.59, 29.3, 25.9, 22.7, 21.0, 19.5, 14.1 ppm. Anal. Calcd for $\mathrm{C}_{43} \mathrm{H}_{78} \mathrm{O}_{3}$ : C, 80.31; H, 12.23; O, 7.46. Found: C, 80.53; H, 12.25; O, 7.49.

1-(Furan-2-yl)-2,2-dimethoxyethanone (5a). ${ }^{7}$ The title compound was prepared via the general procedure from 2acetylfuran $4 \mathbf{a}(110 \mathrm{mg}, 1.0 \mathrm{mmol})$ and methanol $2 \mathbf{b}(1 \mathrm{~mL})$. The product was isolated by flash chromatography using $5 \%$ ethyl acetate/hexane as an eluent; oil (158 mg, 93\% yield); IR(KBr film ): 3138, 2942, 2837, 1688, 1565, 1466, 1396, 1273, 1131, $1066 \mathrm{~cm}^{-1}$; ${ }^{1} \mathrm{H}$ NMR $\left(400 \mathrm{MHz}, \mathrm{CDCl}_{3}\right): \delta 7.60(\mathrm{dd}, J$ $=0.8 \mathrm{~Hz}, 1 \mathrm{H}), 7.38(\mathrm{dd}, J=0.8 \mathrm{~Hz}, 1 \mathrm{H}), 6.49(\mathrm{dd}, J=1.6 \mathrm{~Hz}$, $1 \mathrm{H}), 5.03(\mathrm{~s}, 1 \mathrm{H}), 3.40(\mathrm{~s}, 6 \mathrm{H}) \mathrm{ppm} ;{ }^{13} \mathrm{C}\left\{{ }^{1} \mathrm{H}\right\} \mathrm{NMR}(100$ $\left.\mathrm{MHz}, \mathrm{CDCl}_{3}\right): \delta 181.2,148.9,146.6,120.1,111.2,101.1,53.3$ ppm. MS $\left(\mathrm{ES}^{+}\right)$: for $\mathrm{C}_{8} \mathrm{H}_{10} \mathrm{O}_{4} 170.0$, found $m / z, 193.1[\mathrm{M}+$ $\mathrm{Na}]^{+}$.

2,2-Diethoxy-1-(furan-2-yl)ethanone (5b). ${ }^{9}$ The title compound was prepared via the general procedure from 2acetylfuran $4 \mathrm{a}(110 \mathrm{mg}, 1.0 \mathrm{mmol})$ and ethanol $2 \mathrm{a}(1 \mathrm{~mL})$. The product was isolated by flash chromatography using $2 \%$ ethyl acetate/hexane as an eluent; oil (176 mg, 89\% yield); IR (KBr film): 3139, 2980, 2935, 2887, 1681, 1565, 1465, 1394, 1306, 1268, 1228, 1125, $1061 \mathrm{~cm}^{-1}$; ${ }^{1} \mathrm{H}$ NMR (400 MHz, $\left.\mathrm{CDCl}_{3}\right): \delta 7.59(\mathrm{~s}, 1 \mathrm{H}), 7.43(\mathrm{~d}, J=3.6 \mathrm{~Hz}, 1 \mathrm{H}), 6.49-6.46$ $(\mathrm{m}, 1 \mathrm{H}), 5.08(\mathrm{~s}, 1 \mathrm{H}), 3.73-3.57(\mathrm{~m}, 4 \mathrm{H}), 1.19(\mathrm{t}, J=7.2 \mathrm{~Hz}$, $6 \mathrm{H}) \mathrm{ppm} ;{ }^{13} \mathrm{C}\left\{{ }^{1} \mathrm{H}\right\} \mathrm{NMR}\left(100 \mathrm{MHz}, \mathrm{CDCl}_{3}\right): \delta 183.0,147.4$, 121.2, 112.2, 101.2, 63.0, 15.1 ppm. MS $\left(\mathrm{ES}^{+}\right)$: for $\mathrm{C}_{10} \mathrm{H}_{14} \mathrm{O}_{4}$, 198.1; found $m / z, 199.0[\mathrm{M}+\mathrm{H}]^{+}$.

2,2-Diethoxy-1-(thiophen-2-yl)ethanone (5c). ${ }^{9}$ The title compound was prepared via the general procedure from 2acetylthiophene $4 \mathrm{~b}(126 \mathrm{mg}, 1.0 \mathrm{mmol})$ and ethanol 2a (1 $\mathrm{mL}$ ). The product was isolated by flash chromatography using $2 \%$ ethyl acetate/hexane as an eluent; oil (154 mg, $72 \%$ yield); IR ( $\mathrm{KBr}$ film): 3102, 2979, 2931, 2883, 1670, 1509, 1413, 1363, 1323, 1288, 1240, 1115, $1059 \mathrm{~cm}^{-1} ;{ }^{1} \mathrm{H}$ NMR (400 $\left.\mathrm{MHz}, \mathrm{CDCl}_{3}\right): \delta 8.00(\mathrm{~d}, J=3.6 \mathrm{~Hz}, 1 \mathrm{H}), 7.60(\mathrm{~d}, J=5.2 \mathrm{~Hz}$, $1 \mathrm{H}), 7.07(\mathrm{t}, J=4.8 \mathrm{~Hz}, 1 \mathrm{H}), 5.06(\mathrm{~s}, 1 \mathrm{H}), 3.72-3.57(\mathrm{~m}$, $4 \mathrm{H}), 1.20(\mathrm{t}, J=7.2 \mathrm{~Hz}, 6 \mathrm{H}) \mathrm{ppm} ;{ }^{13} \mathrm{C}\left\{{ }^{1} \mathrm{H}\right\} \mathrm{NMR}(100 \mathrm{MHz}$, $\left.\mathrm{CDCl}_{3}\right): \delta 187.7,139.6,134.8,134.6,128.0,102.2,63.1,15.1$ ppm. MS (ES ${ }^{+}$): for $\mathrm{C}_{10} \mathrm{H}_{14} \mathrm{O}_{3} \mathrm{~S}, 214.0$; found $\mathrm{m} / z, 236.7[\mathrm{M}+$ $\mathrm{Na}]^{+}$.

2,2-Diisobutoxy-1-(thiophen-2-yl)ethanone (5d). The title compound was prepared via the general procedure from 2acetylthiophene $4 \mathrm{~b}(126 \mathrm{mg}, 1.0 \mathrm{mmol})$ and iso-butanol $2 \mathrm{~g}$ (1 $\mathrm{mL}$ ). The product was isolated by flash chromatography using $5 \%$ ethyl acetate/hexane as an eluent; oil $(210 \mathrm{mg}, 78 \%$ yield); IR(KBr film ):3104, 2960, 2874, 1670, 1517, 1414, 1366, 1288, 1124, $1055 \mathrm{~cm}^{-1}$; ${ }^{1} \mathrm{H}$ NMR $\left(400 \mathrm{MHz}, \mathrm{CDCl}_{3}\right.$ ): $\delta 8.00$ (dd, $J$ $=1.2,0.8 \mathrm{~Hz}, 1 \mathrm{H}), 7.60(\mathrm{dd}, J=0.8,1.2 \mathrm{~Hz}, 1 \mathrm{H}), 7.07-7.05$ $(\mathrm{m}, 1 \mathrm{H}), 4.96(\mathrm{~s}, 1 \mathrm{H}), 3.43-3.26(\mathrm{~m}, 4 \mathrm{H}), 1.90-1.80(\mathrm{~m}$, $2 \mathrm{H}), 0.86-0.83(\mathrm{~m}, 12 \mathrm{H}) \mathrm{ppm} ;{ }^{13} \mathrm{C}\left\{{ }^{1} \mathrm{H}\right\} \mathrm{NMR}(100 \mathrm{MHz}$, $\left.\mathrm{CDCl}_{3}\right): \delta 186.8,138.6,133.8,133.4,126.9,102.4,73.4,27.5$, 18.3, 18.2 ppm. MS $\left(\mathrm{ES}^{+}\right)$: for $\mathrm{C}_{14} \mathrm{H}_{22} \mathrm{O}_{3} \mathrm{~S}, 270.1$; found $\mathrm{m} / z$, $293.0[\mathrm{M}+\mathrm{Na}]^{+}$. HRMS (ESI) $m / z:[\mathrm{M}+\mathrm{Na}]^{+}$calcd for $\mathrm{C}_{14} \mathrm{H}_{22} \mathrm{O}_{3} \mathrm{SNa}$, 293.1187; found, 293.1186.
(E)-4-Phenyl-1,1-dipropoxybut-3-en-2-one (7a). ${ }^{4 b}$ The title compound was prepared via the general procedure from benzylidine acetone $6 \mathrm{a}(146 \mathrm{mg}, 1.0 \mathrm{mmol})$ and 1-propanol $2 \mathrm{c}$ $(1 \mathrm{~mL})$. The product was isolated by flash chromatography using 1\% ethyl acetate/hexane as an eluent; oil (225 mg, 86\% yield); IR ( $\mathrm{KBr}$ film): 3061, 3028, 2966, 2935, 2876, 1697, 1610, 1453, 1317, $1070 \mathrm{~cm}^{-1} ;{ }^{1} \mathrm{H}$ NMR (400 MHz, $\left.\mathrm{CDCl}_{3}\right): \delta$ $7.72(\mathrm{~d}, J=16.4 \mathrm{~Hz}, 1 \mathrm{H}), 7.53-7.31(\mathrm{~m}, 5 \mathrm{H}), 7.03(\mathrm{~d}, J=16$ $\mathrm{Hz}, 1 \mathrm{H}), 4.73(\mathrm{~s}, 1 \mathrm{H}), 3.58-3.43(\mathrm{~m}, 4 \mathrm{H}), 1.64-1.55(\mathrm{~m}$, $4 \mathrm{H}), 0.88(\mathrm{t}, J=7.6 \mathrm{~Hz}, 6 \mathrm{H}) \mathrm{ppm} ;{ }^{13} \mathrm{C}\left\{{ }^{1} \mathrm{H}\right\} \mathrm{NMR}(100 \mathrm{MHz}$, $\left.\mathrm{CDCl}_{3}\right): \delta 194.3,144.7,134.6,130.6,128.8,128.5,120.8$, $102.8,69.2,22.9,10.5$ ppm. HRMS (ESI) $m / z:[\mathrm{M}+\mathrm{Na}]^{+}$ calcd for $\mathrm{C}_{16} \mathrm{H}_{22} \mathrm{O}_{3} \mathrm{Na}$, 285.1467; found, 285.1463.

(E)-1,1-Dimethoxy-4-(p-tolyl)but-3-en-2-one (7b). The title compound was prepared via the general procedure from 4-methyl-benzylidine acetone $6 \mathrm{~b}(160 \mathrm{mg}, 1.0 \mathrm{mmol})$ and methanol $\mathbf{2 b}(1 \mathrm{~mL})$. The product was isolated by flash chromatography using $1 \%$ ethyl acetate/hexane as an eluent; oil (202 mg, 92\% yield); IR ( $\mathrm{KBr}$ film):2996, 2935, 2834, 1696, 1602, 1512, 1321, 1183, $1068 \mathrm{~cm}^{-1} ;{ }^{1} \mathrm{H}$ NMR (400 $\left.\mathrm{MHz}, \mathrm{CDCl}_{3}\right): \delta 7.71(\mathrm{~d}, J=16.4 \mathrm{~Hz}, 1 \mathrm{H}), 7.43(\mathrm{~d}, J=8 \mathrm{~Hz}$, $2 \mathrm{H}), 7.13$ (d, $J=8 \mathrm{~Hz}, 2 \mathrm{H}), 6.96$ (d, $J=16 \mathrm{~Hz}, 1 \mathrm{H}), 4.68$ (s, $1 \mathrm{H}), 3.38(\mathrm{~s}, 6 \mathrm{H}), 2.30(\mathrm{~s}, 3 \mathrm{H}) \mathrm{ppm} ;{ }^{13} \mathrm{C}\left\{{ }^{1} \mathrm{H}\right\} \mathrm{NMR}(100$ $\left.\mathrm{MHz}, \mathrm{CDCl}_{3}\right): \delta 192.8,144.2,140.4,130.7,128.6,127.7$, 118.6, 102.6, 53.3, 20.5 ppm. HRMS (ESI) $m / z:[\mathrm{M}+\mathrm{Na}]^{+}$ calcd for $\mathrm{C}_{13} \mathrm{H}_{16} \mathrm{O}_{3} \mathrm{Na}, 243.0997$; found, 243.0995 .

(E)-1,1-Bis(hexyloxy)-4-(4-methoxyphenyl)but-3-en-2-one (7c). The title compound was prepared via the general procedure from 4-methoxy-benzylidine acetone $6 \mathrm{c}(176 \mathrm{mg}$, $1.0 \mathrm{mmol})$ and 1-hexanol $2 \mathrm{e}(1 \mathrm{~mL})$. The product was isolated by flash chromatography using $5 \%$ ethyl acetate/hexane as an eluent; oil (319 mg, 85\% yield); IR ( $\mathrm{KBr}$ film): 2956, 2931, $2863,1694,1596,1512,1463,1256,1174,1066 \mathrm{~cm}^{-1} ;{ }^{1} \mathrm{H}$ NMR (400 MHz, $\left.\mathrm{CDCl}_{3}\right): \delta 7.69(\mathrm{~d}, J=16 \mathrm{~Hz}, 1 \mathrm{H}), 7.48(\mathrm{~d}$, $J=8.4 \mathrm{~Hz}, 2 \mathrm{H}), 6.90(\mathrm{~d}, J=16.4 \mathrm{~Hz}, 1 \mathrm{H}), 6.83(\mathrm{~d}, J=8.8 \mathrm{~Hz}$, $2 \mathrm{H}), 4.71$ (s, $1 \mathrm{H}), 3.77(\mathrm{~s}, 3 \mathrm{H}), 3.61-3.44(\mathrm{~m}, 4 \mathrm{H}), 1.59-$ $1.52(\mathrm{~m}, 4 \mathrm{H}), 1.33-1.19(\mathrm{~m}, 12 \mathrm{H}), 0.80(\mathrm{t}, J=6.8 \mathrm{~Hz}, 6 \mathrm{H})$ ppm; ${ }^{13} \mathrm{C}\left\{{ }^{1} \mathrm{H}\right\} \mathrm{NMR}\left(100 \mathrm{MHz}, \mathrm{CDCl}_{3}\right): \delta$ 194.3, 161.7, 144.5, 130.3, 127.4, 118.5, 114.3, 102.8, 67.5, 55.3, 31.5, 29.6, 25.5, 22.5, $14.0 \mathrm{ppm}$. HRMS (ESI) $m / z:[\mathrm{M}+\mathrm{Na}]^{+}$calcd for $\mathrm{C}_{23} \mathrm{H}_{36} \mathrm{O}_{4} \mathrm{Na}$, 399.2511; found, 399.2515.

(E)-4-(4-Bromophenyl)-1,1-bis(isopentyloxy)but-3-en-2one $(7 d)$. The title compound was prepared via the general procedure from 4-bromo-benzylidine acetone $6 \mathrm{~d}(225 \mathrm{mg}, 1.0$ $\mathrm{mmol})$ and iso-amyl alcohol $2 \mathrm{l}(1 \mathrm{~mL})$. The product was isolated by flash chromatography using $1 \%$ ethyl acetate/ hexane as an eluent; oil ( $353 \mathrm{mg}$, $89 \%$ yield); IR ( $\mathrm{KBr}$ film): 2958, 2872, 1700, 1610, 1486, 1313, 1165, $1068 \mathrm{~cm}^{-1} ;{ }^{1} \mathrm{H}$ NMR (400 MHz, $\left.\mathrm{CDCl}_{3}\right): \delta 7.63(\mathrm{~d}, J=16 \mathrm{~Hz}, 1 \mathrm{H}), 7.45(\mathrm{~d}$, $J=8.8 \mathrm{~Hz}, 2 \mathrm{H}), 7.37(\mathrm{~d}, J=8.4 \mathrm{~Hz}, 2 \mathrm{H}), 6.99(\mathrm{~d}, J=16.4 \mathrm{~Hz}$, $1 \mathrm{H}), 4.68(\mathrm{~s}, 1 \mathrm{H}), 3.67-3.46(\mathrm{~m}, 4 \mathrm{H}), 1.70-1.59(\mathrm{~m}, 2 \mathrm{H})$, $1.48-1.43(\mathrm{~m}, 4 \mathrm{H}), 0.84(\mathrm{~d}, J=2 \mathrm{~Hz}, 6 \mathrm{H}), 0.82(\mathrm{~d}, J=2.4 \mathrm{~Hz}$, $6 \mathrm{H}) \mathrm{ppm} ;{ }^{13} \mathrm{C}\left\{{ }^{1} \mathrm{H}\right\} \mathrm{NMR}\left(100 \mathrm{MHz}, \mathrm{CDCl}_{3}\right): \delta 193.0,142.2$, 132.5, 131.1, 128.8, 123.9, 120.2, 101.9, 65.1, 37.3, 23.9, 21.5 ppm. HRMS (ESI) $m / z:[\mathrm{M}+\mathrm{H}]^{+}$calcd for $\mathrm{C}_{20} \mathrm{H}_{30} \mathrm{BrO}_{3}$, 397.1378; found, 397.1343.

(E)-1,1-Diisopropoxy-4-(4-nitrophenyl)but-3-en-2-one (7e). The title compound was prepared via the general procedure from 4-nitro-benzylidine acetone $6 \mathrm{e}(191 \mathrm{mg}, 1.0$ $\mathrm{mmol})$ and iso-propanol $2 \mathbf{i}(1 \mathrm{~mL})$. The product was isolated by flash chromatography using $5 \%$ ethyl acetate/hexane as an eluent; oil (251 mg, 82\% yield); IR ( $\mathrm{KBr}$ film): 3110, 2974, 2932, 2897, 1700,1616,1519,1345, 1107, $1041 \mathrm{~cm}^{-1} ;{ }^{1} \mathrm{H}$ 
NMR (400 MHz, $\mathrm{CDCl}_{3}$ ): $\delta 8.18(\mathrm{~d}, J=8.8 \mathrm{~Hz}, 2 \mathrm{H}), 7.72-$ $7.66(\mathrm{~m}, 3 \mathrm{H}), 7.14(\mathrm{~d}, J=16 \mathrm{~Hz}, 1 \mathrm{H}), 4.74(\mathrm{~s}, 1 \mathrm{H}), 3.89-3.82$ $(\mathrm{m}, 2 \mathrm{H}), 1.21(\mathrm{~d}, J=6 \mathrm{~Hz}, 6 \mathrm{H}), 1.11(\mathrm{~d}, J=6.4 \mathrm{~Hz}, 6 \mathrm{H}) \mathrm{ppm}$; ${ }^{13} \mathrm{C}\left\{{ }^{1} \mathrm{H}\right\} \mathrm{NMR}\left(100 \mathrm{MHz}, \mathrm{CDCl}_{3}\right): \delta$ 193.0, 147.5, 140.1, 139.9, 128.0, 123.4, 123.0, 99.6, 69.5, 21.9, 21.3 ppm. HRMS (ESI) $m / z:[\mathrm{M}+\mathrm{Na}]^{+}$calcd for $\mathrm{C}_{16} \mathrm{H}_{21} \mathrm{NO}_{5} \mathrm{Na}, 330.1317$; found, 330.1316 .

1,1-Dipropoxypropan-2-one (9a). The title compound was prepared via the general procedure from acetone $8 \mathbf{a}(58 \mathrm{mg}$, $1.0 \mathrm{mmol})$ and 1-propanol $2 \mathrm{c}(1 \mathrm{~mL})$. The product was isolated by column chromatography using $1 \%$ ethyl acetate/ hexane as an eluent; oil (172 mg, 67\% yield); IR ( $\mathrm{KBr}$ film): 2967, 2937, 2879, 1734, 1462, 1381, 1354, 1255, 1111, 1072 $\mathrm{cm}^{-1} ;{ }^{1} \mathrm{H}$ NMR $\left(400 \mathrm{MHz}, \mathrm{CDCl}_{3}\right): \delta 4.44(\mathrm{~s}, 1 \mathrm{H}), 3.56-3.35$ $(\mathrm{m}, 4 \mathrm{H}), 2.13(\mathrm{~s}, 3 \mathrm{H}), 1.61-1.52(\mathrm{~m}, 4 \mathrm{H}), 0.87(\mathrm{t}, J=7.2 \mathrm{~Hz}$, $6 \mathrm{H}) \mathrm{ppm} ;{ }^{13} \mathrm{C}\left\{{ }^{1} \mathrm{H}\right\} \mathrm{NMR}\left(100 \mathrm{MHz}, \mathrm{CDCl}_{3}\right): \delta 203.4,102.0$, 68.4, 23.5, 21.9, 9.5 ppm. HRMS (ESI) $m / z:[\mathrm{M}+\mathrm{Na}]^{+}$calcd for $\mathrm{C}_{9} \mathrm{H}_{18} \mathrm{O}_{3} \mathrm{Na}$, 197.1154; found, 197.1146 .

1,1-Bis(hexyloxy)propan-2-one (9b). The title compound was prepared via the general procedure from acetone 8a (58 $\mathrm{mg}, 1.0 \mathrm{mmol}$ ) and 1 -hexanol $2 \mathrm{e}(1 \mathrm{~mL})$. The product was isolated by column chromatography using $1 \%$ ethyl acetate/ hexane as an eluent; oil (180 mg, 70\% yield); IR ( $\mathrm{KBr}$ film): 2957, 2932, 2865, 1733, 1464, 1353, 1114, $1071 \mathrm{~cm}^{-1} ;{ }^{1} \mathrm{H}$ NMR (400 MHz, $\left.\mathrm{CDCl}_{3}\right): \delta 4.42(\mathrm{~s}, 1 \mathrm{H}), 3.58-3.39(\mathrm{~m}, 4 \mathrm{H})$, $2.12(\mathrm{~s}, 3 \mathrm{H}), 1.57-1.50(\mathrm{~m}, 4 \mathrm{H}), 1.30-1.21(\mathrm{~m}, 12 \mathrm{H}), 0.81$ $(\mathrm{t}, J=6.4 \mathrm{~Hz}, 6 \mathrm{H}) \mathrm{ppm} ;{ }^{13} \mathrm{C}\left\{{ }^{1} \mathrm{H}\right\} \mathrm{NMR}\left(100 \mathrm{MHz}, \mathrm{CDCl}_{3}\right): \delta$ 204.3, 103.0, 67.8, 31.5, 29.5, 25.6, 24.4, 22.5, 13.9 ppm. HRMS (ESI) $m / z:[\mathrm{M}+\mathrm{Na}]^{+}$calcd for $\mathrm{C}_{15} \mathrm{H}_{30} \mathrm{O}_{3} \mathrm{Na}$, 281.2093; found, 281.2084.

1,1-Dipropoxybutan-2-one (9c). The title compound was prepared via the general procedure from ethyl methyl ketone $\mathbf{8 b}(72 \mathrm{mg}, 1.0 \mathrm{mmol})$ and 1-propanol $2 \mathbf{c}(1 \mathrm{~mL})$. The product was isolated by column chromatography using $1 \%$ ethyl acetate/hexane as an eluent; oil (116 mg, 62\% yield); IR ( $\mathrm{KBr}$ film): 2968, 2938, 2878, 1730, 1601, 1461, 1381 1258, 1103, $1074 \mathrm{~cm}^{-1} ;{ }^{1} \mathrm{H}$ NMR $\left(400 \mathrm{MHz}, \mathrm{CDCl}_{3}\right): \delta 4.48(\mathrm{~s}, 1 \mathrm{H})$, $3.55-3.36(\mathrm{~m}, 4 \mathrm{H}), 2.54(\mathrm{q}, J=7.6 \mathrm{~Hz}, 2 \mathrm{H}), 1.60-1.51(\mathrm{~m}$, $4 \mathrm{H}), 0.98(\mathrm{t}, J=7.2 \mathrm{~Hz}, 3 \mathrm{H}), 0.87(\mathrm{t}, J=7.6 \mathrm{~Hz}, 6 \mathrm{H}) \mathrm{ppm}$; ${ }^{13} \mathrm{C}\left\{{ }^{1} \mathrm{H}\right\} \mathrm{NMR}\left(100 \mathrm{MHz}, \mathrm{CDCl}_{3}\right): \delta 207.0,102.9,69.4,30.1$, 22.8, 10.5, 6.9 ppm. HRMS (ESI) $m / z:[\mathrm{M}+\mathrm{Na}]^{+}$calcd for $\mathrm{C}_{10} \mathrm{H}_{20} \mathrm{O}_{3} \mathrm{Na}$, 211.1310; found, 211.1309.

1,1-Diisopropoxypentan-2-one (9d). The title compound was prepared via the general procedure from 2-pentanone $8 \mathrm{c}$ $(86 \mathrm{mg}, 1.0 \mathrm{mmol})$ and iso-propanol $2 \mathrm{i}(1 \mathrm{~mL})$. The product was isolated by column chromatography using $1 \%$ ethyl acetate/hexane as an eluent; oil (137 mg, 68\% yield); IR ( $\mathrm{KBr}$ film): 2971, 2932, 2877, 1726, 1463, 1378, 1104, $1029 \mathrm{~cm}^{-1}$; ${ }^{1} \mathrm{H}$ NMR (400 MHz, $\left.\mathrm{CDCl}_{3}\right): \delta 4.50(\mathrm{~s}, 1 \mathrm{H}), 3.82-3.73(\mathrm{~m}$, $2 \mathrm{H}), 2.51(\mathrm{t}, J=7.2 \mathrm{~Hz}, 2 \mathrm{H}), 1.57-1.48(\mathrm{~m}, 2 \mathrm{H}), 1.16(\mathrm{~d}, J=$ $6.4 \mathrm{~Hz}, 6 \mathrm{H}), 1.08(\mathrm{~d}, J=6 \mathrm{~Hz}, 6 \mathrm{H}), 0.85(\mathrm{t}, J=7.2 \mathrm{~Hz}, 3 \mathrm{H})$ ppm; ${ }^{13} \mathrm{C}\left\{{ }^{1} \mathrm{H}\right\} \mathrm{NMR}\left(100 \mathrm{MHz}, \mathrm{CDCl}_{3}\right): \delta$ 206.0, 99.7, 69.0, 36.6, 21.8, 21.2, 15.4, 12.7 ppm. HRMS (ESI) $m / z:[\mathrm{M}+\mathrm{Na}]^{+}$ calcd for $\mathrm{C}_{11} \mathrm{H}_{22} \mathrm{O}_{3} \mathrm{Na}$, 225.1467; found, 225.1459.

1,1-Dibutoxy-4-methylpentan-2-one (9e). The title compound was prepared via the general procedure from isobutylmethyl ketone $8 \mathrm{~d}$ (100 mg, $1.0 \mathrm{mmol}$ ) and 1-butanol 2d $(1 \mathrm{~mL})$. The product was isolated by column chromatography using 1\% ethyl acetate/hexane as an eluent; oil (168 mg, 69\% yield); IR ( $\mathrm{KBr}$ film): 2960, 2935, 2873, 1729, 1465, 1073 $\mathrm{cm}^{-1}$; ${ }^{1} \mathrm{H} \mathrm{NMR}\left(400 \mathrm{MHz}, \mathrm{CDCl}_{3}\right): \delta 4.41(\mathrm{~s}, 1 \mathrm{H}), 3.58-3.37$ (m, $4 \mathrm{H}), 2.38(\mathrm{~d}, J=6.8 \mathrm{~Hz}, 2 \mathrm{H}), 2.14-2.04(\mathrm{~m}, 1 \mathrm{H}), 1.55-$ $1.48(\mathrm{~m}, 4 \mathrm{H}), 1.36-1.27(\mathrm{~m}, 4 \mathrm{H}), 0.87-0.83(\mathrm{~m}, 12 \mathrm{H}) \mathrm{ppm}$; ${ }^{13} \mathrm{C}\left\{{ }^{1} \mathrm{H}\right\} \mathrm{NMR}\left(100 \mathrm{MHz}, \mathrm{CDCl}_{3}\right): \delta 204.8,102.2,66.5,44.6$, $30.7,22.6,21.5,18.2,12.8$ ppm. HRMS (ESI) $m / z:[\mathrm{M}+\mathrm{Na}]^{+}$ calcd for $\mathrm{C}_{14} \mathrm{H}_{28} \mathrm{O}_{3} \mathrm{Na}$, 267.1936; found, 267.1929.

1,1-Dibutoxy-5-methylhexan-2-one (9f). The title compound was prepared via the general procedure from 5-methyl2-hexanone $8 \mathrm{e}(114 \mathrm{mg}, 1.0 \mathrm{mmol})$ and 1-butanol $2 \mathrm{~d}(1 \mathrm{~mL})$. The product was isolated by column chromatography using $1 \%$ ethyl acetate/hexane as an eluent; oil (172 mg, 67\% yield); IR (KBr film): 2960, 2935, 2873, 1729, 1467, $1074 \mathrm{~cm}^{-1} ;{ }^{1} \mathrm{H}$ NMR (400 MHz, $\left.\mathrm{CDCl}_{3}\right): \delta 4.45(\mathrm{~s}, 1 \mathrm{H}), 3.59-3.37(\mathrm{~m}, 4 \mathrm{H})$, $2.50(\mathrm{t}, J=7.6 \mathrm{~Hz}, 2 \mathrm{H}), 1.55-1.47(\mathrm{~m}, 4 \mathrm{H}), 1.36-1.27(\mathrm{~m}$, $4 \mathrm{H}), 0.87-0.81(\mathrm{~m}, 12 \mathrm{H}) \mathrm{ppm} ;{ }^{13} \mathrm{C}\left\{{ }^{1} \mathrm{H}\right\} \mathrm{NMR}(100 \mathrm{MHz}$, $\left.\mathrm{CDCl}_{3}\right): \delta 205.6,102.1,66.5,44.6,33.7,30.6,26.6,21.3,18.2$, $12.8 \mathrm{ppm}$. HRMS (ESI) $\mathrm{m} / z:[\mathrm{M}+\mathrm{Na}]^{+}$calcd for $\mathrm{C}_{15} \mathrm{H}_{30} \mathrm{O}_{3} \mathrm{Na}$, 281.2093; found, 281.2087.

1,1-Dibutoxy-3-methylbutan-2-one (9g). The title compound was prepared via the general procedure from 3-methyl2-butanone $8 \mathrm{f}$ ( $86 \mathrm{mg}, 1.0 \mathrm{mmol})$ and 1-butanol $\mathbf{2 d}(1 \mathrm{~mL})$. The product was isolated by column chromatography using $1 \%$ ethyl acetate/hexane as an eluent; oil (149 mg, 65\% yield); IR (KBr film): 2963, 2935, 2873, 1727, 1465, 1157, $1074 \mathrm{~cm}^{-1}$; ${ }^{1} \mathrm{H}$ NMR (400 MHz, $\left.\mathrm{CDCl}_{3}\right): \delta 4.57(\mathrm{~s}, 1 \mathrm{H}), 3.58-3.48(\mathrm{~m}$, $4 \mathrm{H}), 3.04-2.93(\mathrm{~m}, 1 \mathrm{H}), 1.56-1.49(\mathrm{~m}, 4 \mathrm{H}), 1.37-1.27(\mathrm{~m}$, $4 \mathrm{H}), 1.02(\mathrm{~d}, J=6.4 \mathrm{~Hz}, 6 \mathrm{H}), 0.85(\mathrm{t}, J=7.2 \mathrm{~Hz}, 6 \mathrm{H}) \mathrm{ppm}$; ${ }^{13} \mathrm{C}\left\{{ }^{1} \mathrm{H}\right\} \mathrm{NMR}\left(100 \mathrm{MHz}, \mathrm{CDCl}_{3}\right): \delta 209.6,102.3,67.3,35.3$, 31.7, 19.2, 18.3, 13.7 ppm. HRMS (ESI) $m / z:[\mathrm{M}+\mathrm{Na}]^{+}$calcd for $\mathrm{C}_{13} \mathrm{H}_{26} \mathrm{O}_{3} \mathrm{Na}$, 253.1780; found, 253.1774.

2,2-Dibutoxy-1-cyclopropylethanone (9h). The title compound was prepared via the general procedure from cyclopropyl methyl ketone $8 \mathrm{~g}$ (84 $\mathrm{mg}, 1.0 \mathrm{mmol})$ and 1-butanol 2d ( $1 \mathrm{~mL})$. The product was isolated by column chromatography using 1\% ethyl acetate/hexane as an eluent; oil (166 mg, 73\% yield); IR (KBr film): 3009, 2960, 2935, 2873, 1713, 1462, 1385, 1161, $1070 \mathrm{~cm}^{-1}$; ${ }^{1} \mathrm{H}$ NMR (400 MHz, $\mathrm{CDCl}_{3}$ ): $\delta 4.61$ (s, $1 \mathrm{H}), 3.59-3.44(\mathrm{~m}, 4 \mathrm{H}), 2.32-2.26(\mathrm{~m}, 1 \mathrm{H}), 1.58-1.51$ $(\mathrm{m}, 4 \mathrm{H}), 1.38-1.29(\mathrm{~m}, 4 \mathrm{H}), 1.02-0.98(\mathrm{~m}, 2 \mathrm{H}), 0.91-0.84$ (m, 8H) ppm; ${ }^{13} \mathrm{C}\left\{{ }^{1} \mathrm{H}\right\} \mathrm{NMR}\left(100 \mathrm{MHz}, \mathrm{CDCl}_{3}\right): \delta$ 205.3, $101.6,66.1,30.7,18.2,15.2,12.8,10.8$ ppm. HRMS (ESI) $m /$ $z:[\mathrm{M}+\mathrm{Na}]^{+}$calcd for $\mathrm{C}_{13} \mathrm{H}_{24} \mathrm{O}_{3} \mathrm{Na}, 251.1623$; found, 251.1614 .

\section{ASSOCIATED CONTENT}

\section{Supporting Information}

The Supporting Information is available free of charge on the ACS Publications website at DOI: 10.1021/acsomega.9b00361.

$$
\text { Copies of }{ }^{1} \mathrm{H} \text { NMR and }{ }^{13} \mathrm{C} \text { NMR spectra (PDF) }
$$

\section{AUTHOR INFORMATION}

\section{Corresponding Author}

*E-mail: bmyrboh@nehu.ac.in.

ORCID ${ }^{\circ}$

Bekington Myrboh: 0000-0001-9349-2216

Notes

The authors declare no competing financial interest.

\section{ACKNOWLEDGMENTS}

The authors thank SAIF-NEHU, SAIF-CDRI, Lucknow, and University of Southampton for spectral analyses. O.R.S. thanks the University Grants Commission (UGC), India, for the 
financial assistance under the National Fellowship for Higher Education (NFHE) Scheme. B.M. acknowledges the financial assistance from SERB, DST (SB/EMEQ-006/2013).

\section{REFERENCES}

(1) (a) Cho, B. T.; Chun, Y. S. Asymmetric reduction of $\alpha$-keto acetals with potassium 9-O-(1,2-isopropylidene-5-deoxy-D-xylofuranosyl)-9-boratabicyclo[3.3.1] nonane. Enantioselective synthesis of $\alpha$ hydroxy acetals with high optical purities. Tetrahedron: Asymmetry 1994, 5, 1147-1150. (b) Studer, M.; Burkhardt, S.; Blaser, H.-U. Enantioselective hydrogenation of $\alpha$-keto acetals with cinchona modified Pt catalyst. Chem. Commun. 1999, 1727-1728. (c) Cho, B. T.; Chun, Y. S. Catalytic enantioselective reactions. Part 16. Oxazaborolidine-catalyzed asymmetric borane reduction of $\alpha$-keto acetals. J. Chem. Soc., Perkin Trans. 1 1999, 2095-2100. (d) Wu, H.; Wang, Q; Zhu, J. Organocatalytic Enantioselective Acyloin Rearrangement of $\alpha$-Hydroxy Acetals to $\alpha$-Alkoxy Ketones. Angew. Chem., Int. Ed. 2017, 56, 5858-5861. (e) Xue, X.; Chen, P.; Xu, P.; Wang, Y. Highly Efficient and Recyclable Chiral Pt Nanoparticle Catalyst for Enantioselective Hydrogenation of Activated Ketones. Catal. Commun. 2018, 110, 55-58.

(2) Pan, H.; Xie, Y.; Liu, M.; Shi, Y. Organocatalytic asymmetric biomimetic transamination of $\alpha$-keto acetals to chiral $\alpha$-amino acetals. RSC Adv. 2014, 4, 2389-2392.

(3) (a) Akhoon, K. M.; Myles, D. C. Highly Diastereoselective Additions to Chiral $\alpha$-Keto Acetals. J. Org. Chem. 1997, 62, 60416045. (b) Becerra-Martínez, E.; Velázquez-Ponce, P.; SánchezAguilar, M. A.; Rodríguez-Hosteguín, A.; Joseph-Nathan, P.; Tamariza, J.; Zepeda, L. G. New 2-acyl-1, 3-dioxane Derivatives from (1R) -(-)-myrtenal: Stereochemical Effect on their Relative Ability as Chiral Auxiliaries. Tetrahedron: Asymmetry 2007, 18, 27272737. (c) Vargas-Díaz, M. E.; Mendoza-Figueroa, H. L.; FragosoVázquez, M. J.; Ayala-Mata, F.; Joseph-Nathan, P.; Zepeda, L. G. Synthesis of acyldodecaheterocycles derived from (1R)-(-)-myrtenal and evaluation as chiral auxiliaries. Tetrahedron: Asymmetry 2012, 23, $1588-1595$.

(4) (a) Tian, S.-K.; Deng, L. A Highly Enantioselective Chiral Lewis Base-Catalyzed Asymmetric Cyanation of Ketones. J. Am. Chem. Soc. 2001, 123, 6195-6196. (b) Tian, S.-K.; Hong, R.; Deng, L. Catalytic Asymmetric Cyanosilylation of Ketones with Chiral Lewis Base. J. Am. Chem. Soc. 2003, 125, 9900-9901. (c) Qin, B.; Liu, X.; Shi, J.; Zheng, K.; Zhao, H.; Feng, X. Enantioselective Cyanosilylation of $\alpha, \alpha$ Dialkoxy Ketones Catalyzed by Proline-Derived in-Situ-PreparedNOxide as Bifunctional Organocatalyst. J. Org. Chem. 2007, 72, 23742378.

(5) (a) Goswami, S.; Maity, A. C.; Fun, H.-K.; Chantrapromma, S. The Smallest Vicinal Tricarbonyl Compound as a Monohydrate and Tetracarbonyl Compound as a Thiane Derivative - First Effective Synthesis, Characterization and Chemistry. Eur. J. Org. Chem. 2009, 2009, 1417-1426. (b) Goswami, S.; Hazra, A.; Jana, S.; Fun, H.-K. Recognition Study of Substituted Benzoic Acids by 7-Substituted Pterin Receptors in Solution and Solid Phases. CrystEngComm 2010, 12, 1501-1508. (c) Nes, I.; Sydnes, L. K. Formation of NHeterocycles from 1, 1-Diethoxy-5-hydroyalk-3-yn-2-ones. Synthesis 2015, 47, 89-94.

(6) (a) Adamczyk, M.; Johnson, D. D.; Mattingly, P. G.; Pan, Y.; Reddy, R. E. A Convenient Method for the Preparation of $\alpha$-Keto Acetals. Synth. Commun. 2002, 32, 3199-3205. (b) Verhé, R.; Courtheyn, D.; de Kimpe, N.; de Buyck, L.; Schamp, N. Preparation of 1,1-Dialkoxy-2-alkanones. Synthesis 1982, 667-670. (c) Tiecco, M.; Testaferri, L.; Tingoli, M.; Chianelli, D.; Bartoli, D. Seleniummediated conversion of alkynes into .alpha.-dicarbonyl compounds. $J$. Org. Chem. 1991, 56, 4529-4534. (d) Yu, Y.; Chen, G.-q.; Zhu, J.; Zhang, X.-s.; Chen, S.-x.; Tang, H.-t.; Zhang, P. A study of rearrangement of some 1,3-dimethoxyalkan-2-ones. J. Chem. Soc., Perkin Trans. 1 1990, 2239-2243. (e) Samant, S.; Jadhav, B. Unusual Tandem Oxidative C-C Bond Cleavage and Acetalization of Chalcone Epoxides in the Presence of Iodine in Methanol. Synlett 2014, 25,
1591-1595. (f) Liu, X.; Xu, H.; Ma, Z.; Zhang, H.; Wu, C.; Liu, Z. $\mathrm{Cu}$-catalyzed aerobic oxygenation of 2-phenoxyacetophenones to alkyloxy acetophenones. RSC Adv. 2016, 6, 27126-27129.

(7) Tiecco, M.; Testaferri, L.; Tingoli, M.; Bartoli, D. Seleniumcatalyzed conversion of methyl ketones into alpha.-keto acetals. J. Org. Chem. 1990, 55, 4523-4528.

(8) Ayala-Mata, F.; Barrera-Mendoza, C.; Jiménez-Vázquez, H.; Vargas-Díaz, E.; Zepeda, L. Efficient Preparation of $\alpha$-Ketoacetals. Molecules 2012, 17, 13864-13878.

(9) Kharkongor, I.; Myrboh, B. One-pot synthesis of $\alpha$-ketoacetals from aryl methyl ketones in the presence of selenous acid catalyzed by boron trifluoride etherate. Tetrahedron Lett. 2015, 56, 4359-4362.

(10) Laloo, B. M.; Mecadon, H.; Rohman, M. R.; Kharbangar, I.; Kharkongor, I.; Rajbangshi, M.; Nongkhlaw, R.; Myrboh, B. Reaction of Selenium Dioxide with Aromatic Ketones in the presence of Boron Triflouride Etherate: a Protocol for the Synthesis of Triarylethanones. J. Org. Chem. 2012, 77, 707-712.

(11) (a) Myrboh, M. R.; Kharkongor, I.; Rajbangshi, M.; Mecadon, H.; Laloo, B. M.; Sahu, P. R.; Kharbangar, I.; Myrboh, B. One-Pot Synthesis of Unsymmetrical Benzils by Oxidative Coupling using Selenium Dioxide and p-Toluenesulfonic Acid Monohydrate. Eur. J. Org. Chem. 2012, 2012, 320-328. (b) Kharkongor, I.; Rohman, M. R.; Myrboh, B. One-Pot Synthesis of Unsymmetrical Benzils from Aryl Methyl Ketones and Arenes in the Presence of Selenous Acid Catalysed by p-Toluenesulfonic Acid Monohydrate. Tetrahedron Lett. 2012, 53, 2837-2841. (c) Shangpliang, O. R.; Kshiar, B.; Wanniang, K.; Marpna, I. D.; Lipon, T. M.; Laloo, B. M.; Myrboh, B. Selenium Dioxide As an Alternative Reagent for the Direct $\alpha$-Selenoamidation of Aryl Methyl Ketones. J. Org. Chem. 2018, 83, 5829-5835. 